\title{
High Performance Opening Up Dual-core Photonic Crystal Fiber Sensor Based on Surface Plasmon Resonance
}

\section{Soghra Ghahramani}

Tabriz University: University of Tabriz

Jamal Bravestani ( $\sim$ barvestani@tabrizu.ac.ir)

University of Tabriz https://orcid.org/0000-0002-2009-2964

\section{Bahar Meshginqalam}

Tabriz University: University of Tabriz

\section{Research Article}

Keywords: photonic crystal fiber, dual-core sensor, surface plasmon resonance, sensitivity, FDTD

Posted Date: May 19th, 2021

DOl: https://doi.org/10.21203/rs.3.rs-489604/v1

License: (1) This work is licensed under a Creative Commons Attribution 4.0 International License.

Read Full License

Version of Record: A version of this preprint was published at Plasmonics on August 2nd, 2021. See the published version at https://doi.org/10.1007/s11468-021-01513-7. 


\title{
High performance opening up dual-core photonic crystal fiber sensor based on surface plasmon resonance
}

\author{
Ghahramani Soghra, Barvestani Jamal*, Meshginqalam Bahar \\ Faculty of Physics, University of Tabriz, Tabriz, Iran \\ "barvestani@ tabrizu.ac.ir
}

\section{Abstract:}

Nowadays, plasmonic sensor based on photonic crystal fiber (PCF) attracted a great deal of attention in field of optical sensing. An opening up dual-core photonic crystal fiber based on surface plasmon resonance (SPR) are numerically demonstrated and analyzed for detecting wide refractive index (RI) range by Finite-Difference TimeDomain method (FDTD). The wavelength and amplitude integration methods, as well as figure of merit are used to investigate the sensor performance. For improving sensing performance, it is introduced a large hole between two cores in opening up section. The opening up section as a sensing channel is coated with gold film and a thin titanium dioxide $\left(\mathrm{TiO}_{2}\right)$ layer. By surface engineering including imposing of grating on the gold film, specification of optimized values of different layers located near the surface, sensing performance are investigated. Next, the effect of the fiber structural parameters is analyzed to enhancing of SPR and fundamental core mode coupling. The proposed sensor revealed maximum wavelength and amplitude sensitivities of $15167\left(\frac{n m}{R I U}\right)$ and $207.19\left(R I U^{-1}\right)$, respectively. Due to ease of infiltering analyte and gold coating and tanks to high wavelength and amplitude sensitivity, the sensors can be promising candidate of physical and chemical sensing.

Keyword: photonic crystal fiber, dual-core sensor, surface plasmon resonance, sensitivity, FDTD

\section{Introduction}

Surface plasmon resonance (SPR) refers to an electromagnetic phenomenon, which is generated by the combination of free electron oscillations and a transverse magnetic polarized electromagnetic wave on the surface between dielectric medium and metal film [1,2]. Due to outstanding features such as label-free monitoring, high sensitivity, and realtime detection, as well as owning multifarious application such as environmental monitoring, medical diagnostics and food safety, polarization filters, and absorbers, SPR has achieved unprecedented progress in the realm of sensors [24]. Kretschman proposed a configuration of SPR sensor based on prism coupling [2, 3, 5]. Bulky apparatus, heavy weight, inability of remote detection, and inflexibility were major inefficiencies of this configuration, which refined by advent of photonic crystal fiber SPR sensors by Jorgenson where gold was used to create the SPR phenomenon [25]. Owning outstanding characteristics such as tunable effective refractive index in the fiber core, controllable birefringence, and superior light confining capabilities, PCF-SPR sensors have drawn a great deal of attentions [2, 4]. SPR based sensors, according to PCF's properties, use several sensing configurations mainly including internal and external metal coating-based sensing approaches. In internal sensing, analyte is infiltrated in the selective micro-meter sized air holes [6,7]. Rifat et al. proposed a SPR-PCF sensor in which introduced a large air-hole beside the core for efficient light coupling between the cores and SPR modes [8]. In addition, this large air hole will facilitate material coating and effective analyte flow. Conversely, external metal coating-based sensing method is commonly used; Not only does it provide more flexibility, but it is easier compared to covering the inner air-holes as well [8,9]. To date, various externally coated SPR based PCF sensors, which can include D-shaped structures, have been reported. A dualpolarized spiral photonic crystal fiber based on surface plasmon resonance was proposed in ref. [9]. They showed wavelength sensitivity of $4600 \frac{\mathrm{nm}}{R I U}$ and amplitude sensitivity of $420.4 R I U^{-1}$ in y-polarized mode. In the x-polarized mode, the maximum wavelength sensitivity is $4300 \frac{\mathrm{nm}}{R I U}$ and amplitude sensitivity is $371.5 \mathrm{RIU}^{-1}$. A dual-core PCF sensor using gold as a plasmonic material with high value of amplitude sensitivity but lower value of wavelength sensitivity was reported by Paul et al. [10]. In 2020, designed a large detection-range plasmonic sensor based on a Hshaped PCF with maximum wavelength sensitivity of $25900 \frac{\mathrm{nm}}{R I U}$ [11]. Recently, SPR based PCF sensors encounter with two main problems. First, due to micro-sized air holes, metal coating and analyte filling are challenging procedures $[8,9,11,12]$. Second problem is about low RI or high RI PCF-SPR sensors due to their narrow RI range 
of detection $[6,11]$. This problem can be solved with respect to the photonic crystal fiber uses the total internal refraction either the crystal geometric properties for light confinement.

Opening up microstructured optical fiber (MOF) structures, such as D-shaped or exposed-core MOF-SPR sensors [13-15] are the promising approaches to evade of infiltrate analyte. Opening up dual-core microstructure optical fiberbased plasmonic sensor with large detection range and linear sensitivity is proposed in [14] and showed the maximal sensitivity of $4900 \frac{n m}{R I U}$ when the RI of the analyte is close to that of the fiber background material. To overcome the problems aforementioned, in this paper we propose an opening up plasmonic sensor based on dual-core PCF which can be operated in range from 1.42 to 1.46 . For improving sensing performance, it is introduced a large hole between two cores in opening up slot. The opening up section as a sensing channel is coated with gold film where a thin titanium dioxide $\left(\mathrm{TiO}_{2}\right)$ layer is placed between gold and analyte in only this hole part. Next, with imposing changes in surface of this part including applying grated gold film and sandwiching thin layer of $\mathrm{TiO}_{2}$ between fiber surface and gold, we investigate effect of these structure variations on sensing performance. Recently, investigation revealed that gold layer coated on fiber can be flaked off from the fiber [6]. $\mathrm{TiO}_{2}$ accompanied by gold layer can conquer the adhesion problem of gold to fiber [16]. Regard to sensitivity, this sensor shows high sensitivity value better than that of [14]. The opening up part directly fills with analyte and supports possibility for real-time sensing.

\section{Design and numerical method}

Figure 1(a) illustrates the schematic representation of our proposed sensor, comprises open slot which is coated by a gold layer. This part acts as a sensing channel and can be directly in contact with analyte. In addition, to improve the sensing performance, we introduce a large hole with diameter of $2 r_{c}$ between two cores in sensing channel. Since, evanescent waves can enhance resonance effect, which may improve the sensitivity significantly. This dual-core SPRPCF sensor has been designed by arranging the air-holes in hexagonal lattice with pitch size of $\Lambda$. These air-holes with radius of $r$ work as a low refractive index cladding, enabling mode guidance in the fiber core. Furthermore, we applied a shift by a distance $d x$ in the center of four selected air holes which is situated neighborhood of the large hole from their original position. Shifting holes lead to achieve optimum structure which is needed to get high sensitivity. With the purpose of increasing the plasmon excitation, a thin layer of $\mathrm{TiO}_{2}$ with the thickness of $t_{\mathrm{TiO}_{2}}$ is deposited on gold.

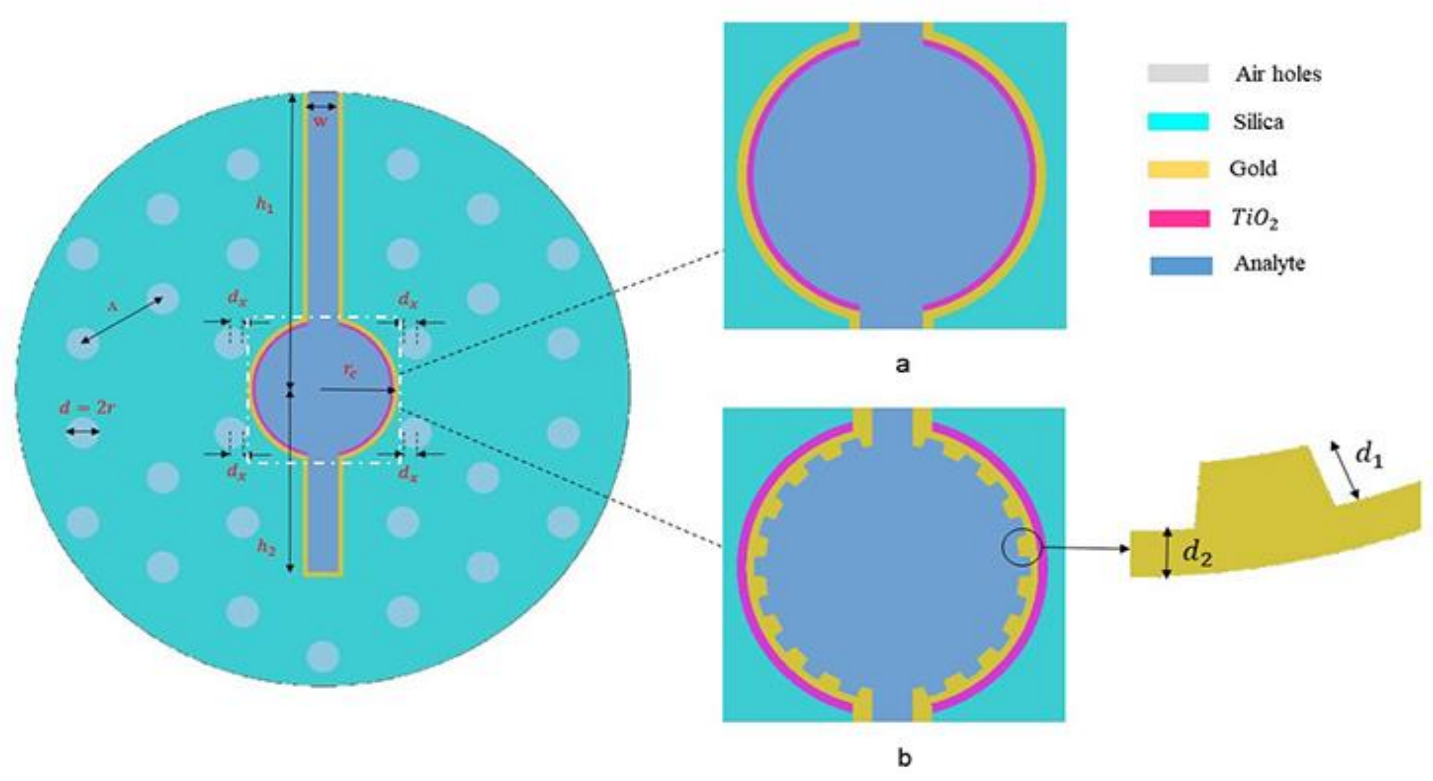


Fig. 1 Cross-section view of DC-PCF-SPR sensor magnifying of central large hole a with $\mathrm{Au}$ and $\mathrm{TiO}_{2}$ thin layer between $\mathrm{Au}$ and analyte $\mathbf{b}$ with $\mathrm{Au}$ nano-continues gratings layer and thin $\mathrm{TiO}_{2}$ layer between fiber material and $\mathrm{Au}$

Our sensor is modeled using the following parameters; $\Lambda=2.26 \mu \mathrm{m}, r=0.4 \mu \mathrm{m}, d x=0.3 \mu \mathrm{m}, r_{c}=1.85 \mu \mathrm{m}$, $t_{A u}=40 \mathrm{~nm}, t_{\text {TiO }_{2}}=7 \mathrm{~nm}, w=1 \mu \mathrm{m}, h_{1}=7.5 \mu \mathrm{m}, h_{2}=4.75 \mu \mathrm{m}$. The refractive indices of background material and air-holes are supposed to be 1.45 and 1 respectively, and gold permittivity is modelled from Johnson and Christy data [17]. The RI of $\mathrm{TiO}_{2}$ is calculated by [18]:

$n_{\text {Tio }_{2}}^{2}=5.913+\frac{0.2441}{\lambda^{2}-0.0803}$,

$\lambda$ is in $\mu$ m unit.

Perfectly matched layer (PML) is applied as scattering boundary condition. FDTD method is employed to investigate the sensor performance. Figure 1(b) shows the same structure associated with some changes in the surface of the proposed sensor. Indeed grated $\mathrm{Au}$ is used as a plasmonic material in large hole part and $\mathrm{TiO}_{2}$ thin layer is deposited between fiber and gold due to assist adhesion. The optimized parameters are as follows: segmented $A u$ film thickness $d_{1}=25 \mathrm{~nm}$ and continuous $A u$ films thickness $d_{2}=15 \mathrm{~nm}$, total segment number $N=28, t_{\mathrm{TiO}_{2}}=5 \mathrm{~nm}$. Other geometric parameters are the same as mentioned before.

The key factor to analyze the performance of PCF-SPR sensors is calculation of the confinement loss of the fundamental core mode. The imaginary part of the effective refractive index $\left(n_{\text {eff }}\right)$ is used to determine the confinement loss and can be expressed as [19]:

$\alpha_{c}\left(\frac{d B}{c m}\right)=8.686 \times \frac{2 \pi}{\lambda(\mu m)} \operatorname{Im}\left(n_{e f f}\right) \times 10^{4}$,

where $\lambda$ is the operating wavelength. The proposed sensor has two guiding modes (a) x-polarization and (b) $y$ polarization. In the dual-core PCF-SPR sensors, for x-polarization and y-polarization, the odd and even modes are excited simultaneously. But here the confinement loss of the odd mode for y-polarization is the largest, which means that SPR mode couples with odd mode for y-polarization more strongly than the other polarization. Hence, we focus on odd core mode for y-polarization in the following numerical analysis.

\section{Results and discussion}

Different structural parameters such as radius of central large hole $\left(r_{c}\right)$, width of slot $(w)$, distance between center to end of slot $\left(h_{2}\right)$ and the position of the neighboring holes of central hole $(d x)$ are examined and optimum parameters are selected throughout this work. Performance of the proposed sensor is numerically carried out by FDTD method in wavelength range of 0.975-1.7 $\mu \mathrm{m}$ and fundamental core mode, SPR mode and dispersion relation are investigated for the proposed sensor. First part of results is associated with the configuration of figure 1(a). The electric field profile of odd fundamental core, SPR and coupled core-SPR modes at resonance wavelength are depicted in figure 2(a)-(c), respectively. Obviously, in resonance condition most of the energy is confined in core regions, and only a small part of energy penetrates to metal film surface, see panel (c). This penetration causes a peak in loss spectrum which can be analyzed by the dispersion relationship between the fundamental core mode and SPR mode as it is shown in panel (d). In fact, coupling between the core and plasmonic modes occurs when propagation constant and wavevector of two modes become equal. This condition is known as phase matching. 


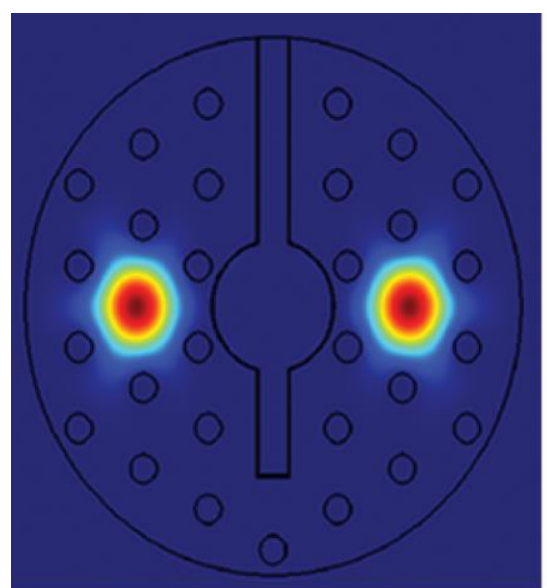

a

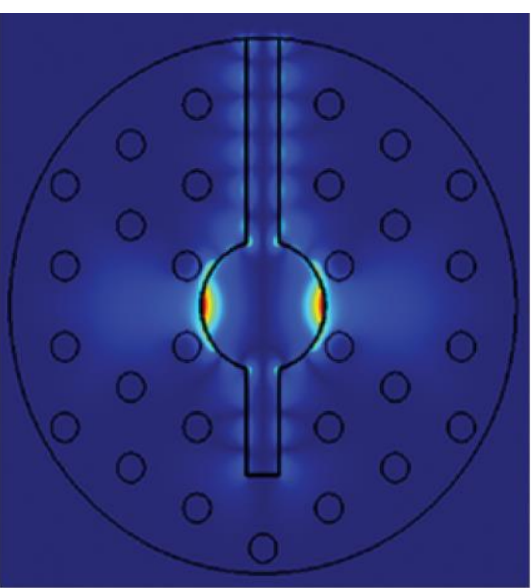

b

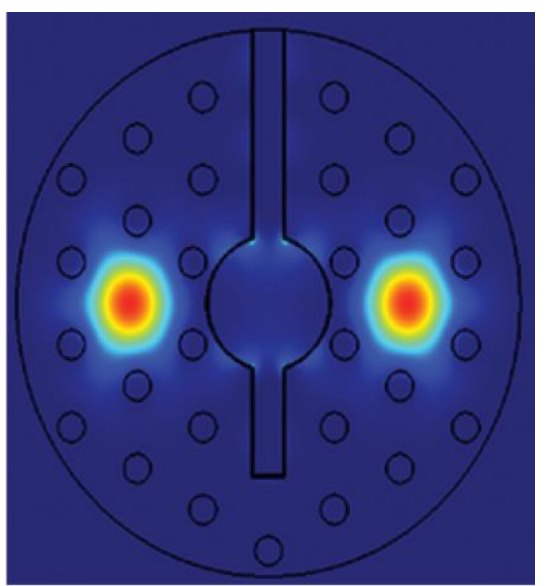

C

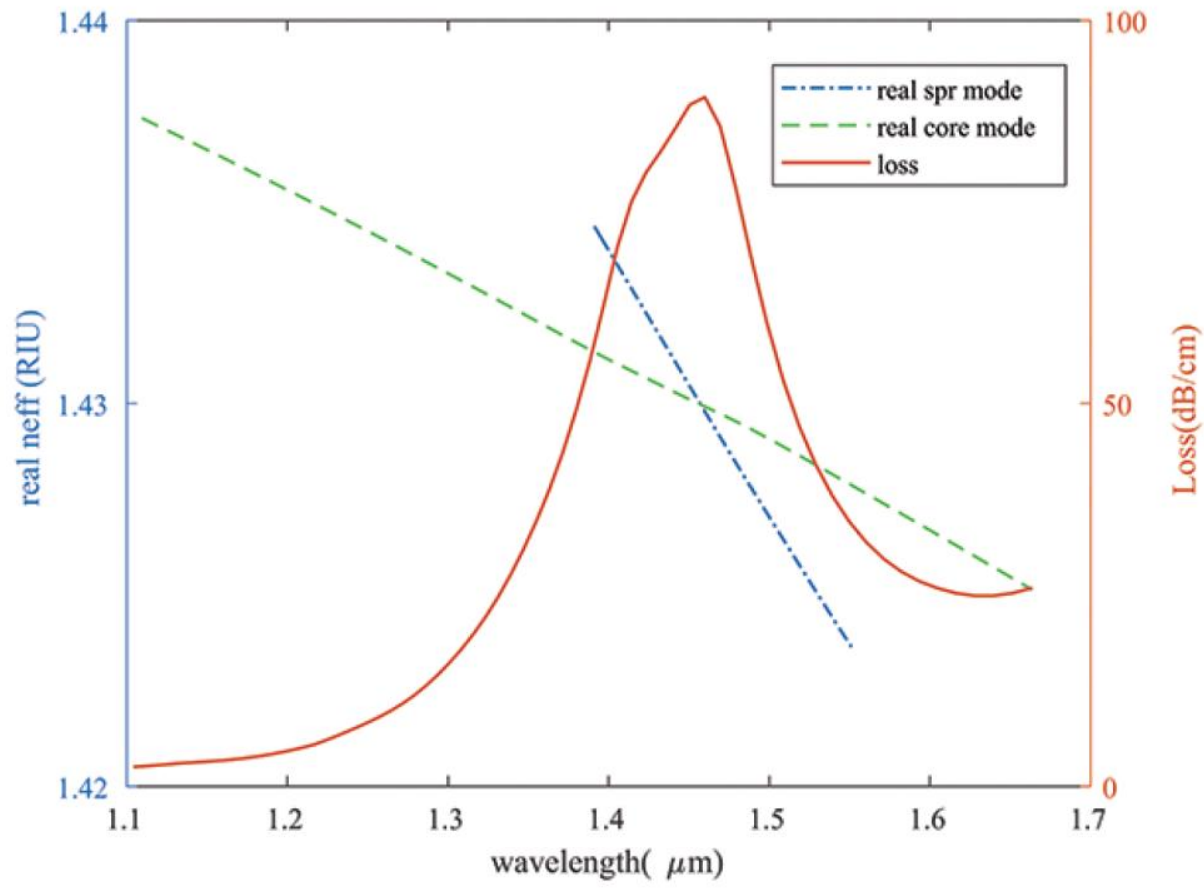

d

Fig. 2 Electric field distribution of the $\mathbf{a}$ y-polarized odd core mode, $\mathbf{b}$ odd SPP mode, $\mathbf{c}$ resonance condition, and $\mathbf{d}$ dispersion relation of core mode (green), SPR mode (blue), and loss spectrum (red) of core mode for $n_{a}=1.43$, $t_{\mathrm{TiO}_{2}}=7 \mathrm{~nm}, t_{\mathrm{Au}}=40 \mathrm{~nm}$

As it is clear from this panel, the real part of $n_{\text {eff }}$ of both modes coincides at the wavelength of $1.4589 \mu \mathrm{m}$, called resonance wavelength where corresponding loss is $89.97 \frac{\mathrm{dB}}{\mathrm{cm}}$. 
The real part of the effective index of the plasmonic mode is highly dependent on the small variation of analyte RI. When RI of analyte is changed, it leads to the resonance wavelength shifts. Using the mentioned optimized parameters, the loss curves of the proposed dual-core SPR-PCF sensor for different RI of analyte ranging from 1.42 to 1.46 in the absence of $\mathrm{TiO}_{2}$ layer are plotted and shown in Figure 3.

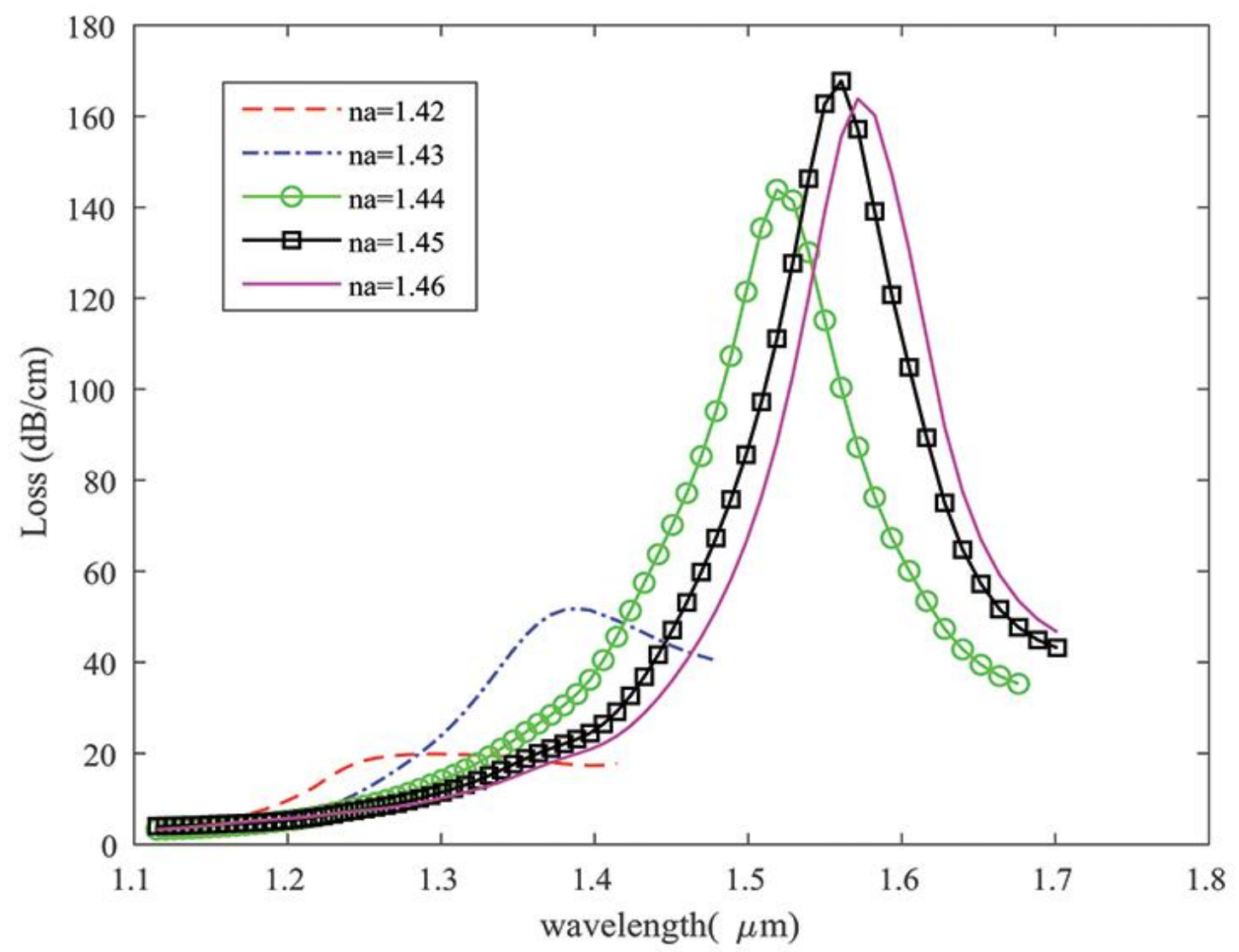

Fig. 3 Loss curves as a function of operating wavelength of the proposed sensor for different analyte for without $\mathrm{TiO}_{2}$ configuration $\left(t_{\mathrm{TiO}_{2}}=0 \mathrm{~nm}\right), t_{\mathrm{Au}}=40 \mathrm{~nm}$

As it is clear from the figure 3, with increasing $n_{a}$ up to 1.45 , red shift of resonance wavelengths is found, and the loss spectra noticeably increases. But, when $n_{a}$ changes from 1.45 to 1.46 , resonance wavelength shifts towards longer wavelength, while loss decreases. Next, we have examined the loss curves of proposed sensor by introducing extra over layer of $\mathrm{TiO}_{2}$. Interestingly it can be observed that by applying $\mathrm{TiO}_{2}$ layer, monotonic increasing trend in resonance wavelength and its intensity is achieved, as it is shown in figure 4. 


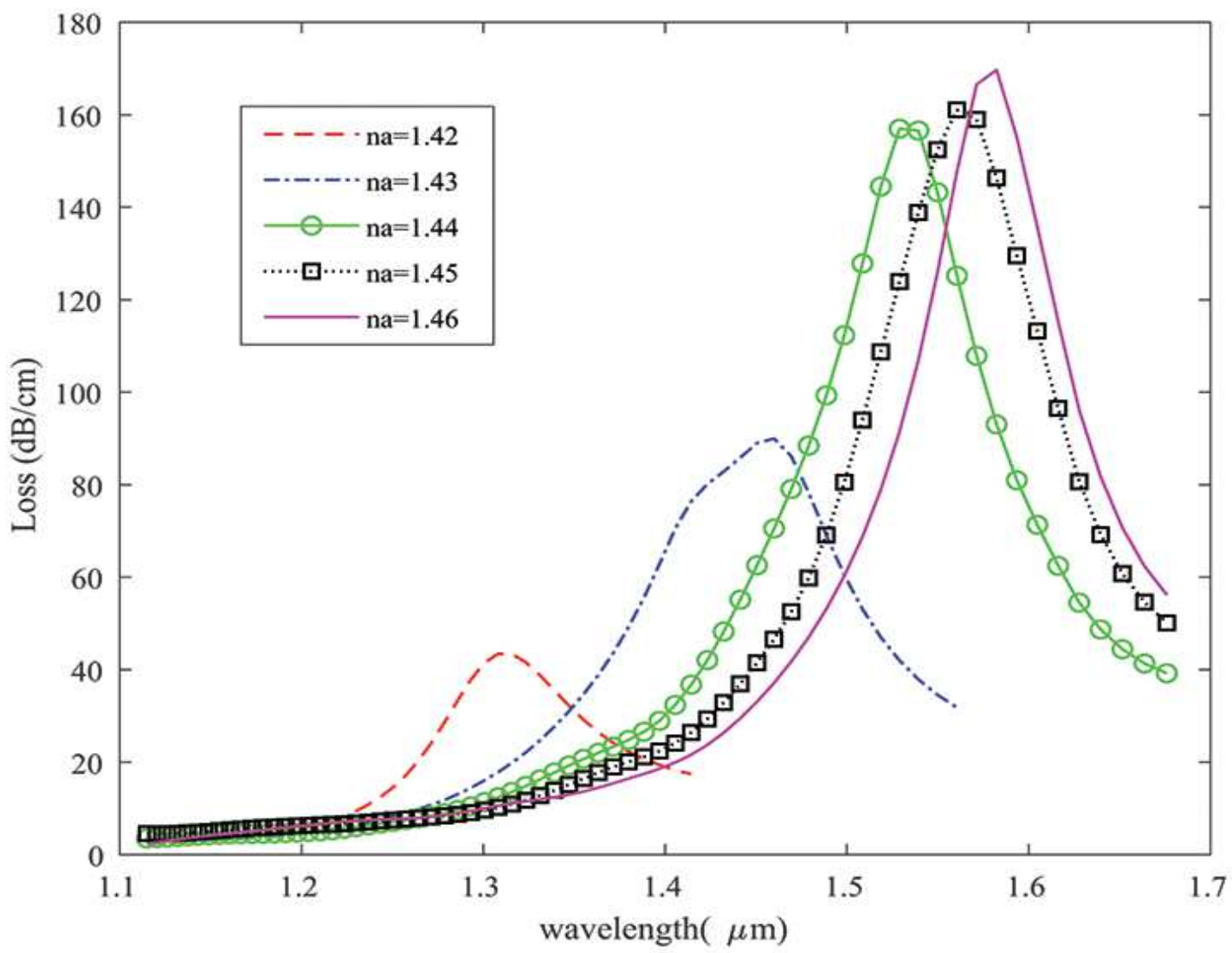

Fig. 4 Loss curves as a function of operating wavelength of the proposed sensor for different analyte for $t_{\mathrm{TiO}_{2}}=$

$$
7 \mathrm{~nm}, t_{A u}=40 \mathrm{~nm}
$$

With increasing RI of analyte $n_{a}$, the peak of loss shifts toward longer wavelength and confinement loss increases, too.

It is convenient to investigate the sensor performance from the loss curve by using the wavelength and amplitude interrogation methods. The ratio of peak wavelength change to refractive index is known as the wavelength sensitivity and it is computed as bellows [20]:

$S_{\lambda}=\frac{\partial \lambda_{\text {peak }}}{\partial n_{a}}\left[\frac{n m}{R I U}\right]$.

Also, the amplitude sensitivity can be evaluated by the following equation [20]:

$S_{A}=-\frac{1}{\alpha\left(\lambda, n_{a}\right)} \times \frac{\partial \alpha\left(\lambda, n_{a}\right)}{\partial n_{a}} \quad\left[R I U^{-1}\right]$

where $\alpha\left(\lambda, n_{a}\right)$ is the confinement loss at different RI. With these definitions, the proposed sensor shows a wavelength sensitivities of $15167,6894,3158$ and $2179 \frac{\mathrm{nm}}{R I U}$, respectively, when the analyte' s RI changes from 1.42 to 1.46 with a step of 0.01 . The maximum wavelength sensitivity is $15167 \frac{\mathrm{nm}}{\mathrm{RIU}}$ which is higher than that of previously proposed in [14] which is a similar work and also it is higher than the maximum wavelength sensitivity of proposed sensor in absence of $\mathrm{TiO}_{2}\left(13024 \frac{\mathrm{nm}}{\mathrm{RIU}}\right)$. Furthermore, the amplitude sensitivities are obtained 207.19, 62.55, 35.40, and 22.91 $R I U^{-1}$, correspondingly. 


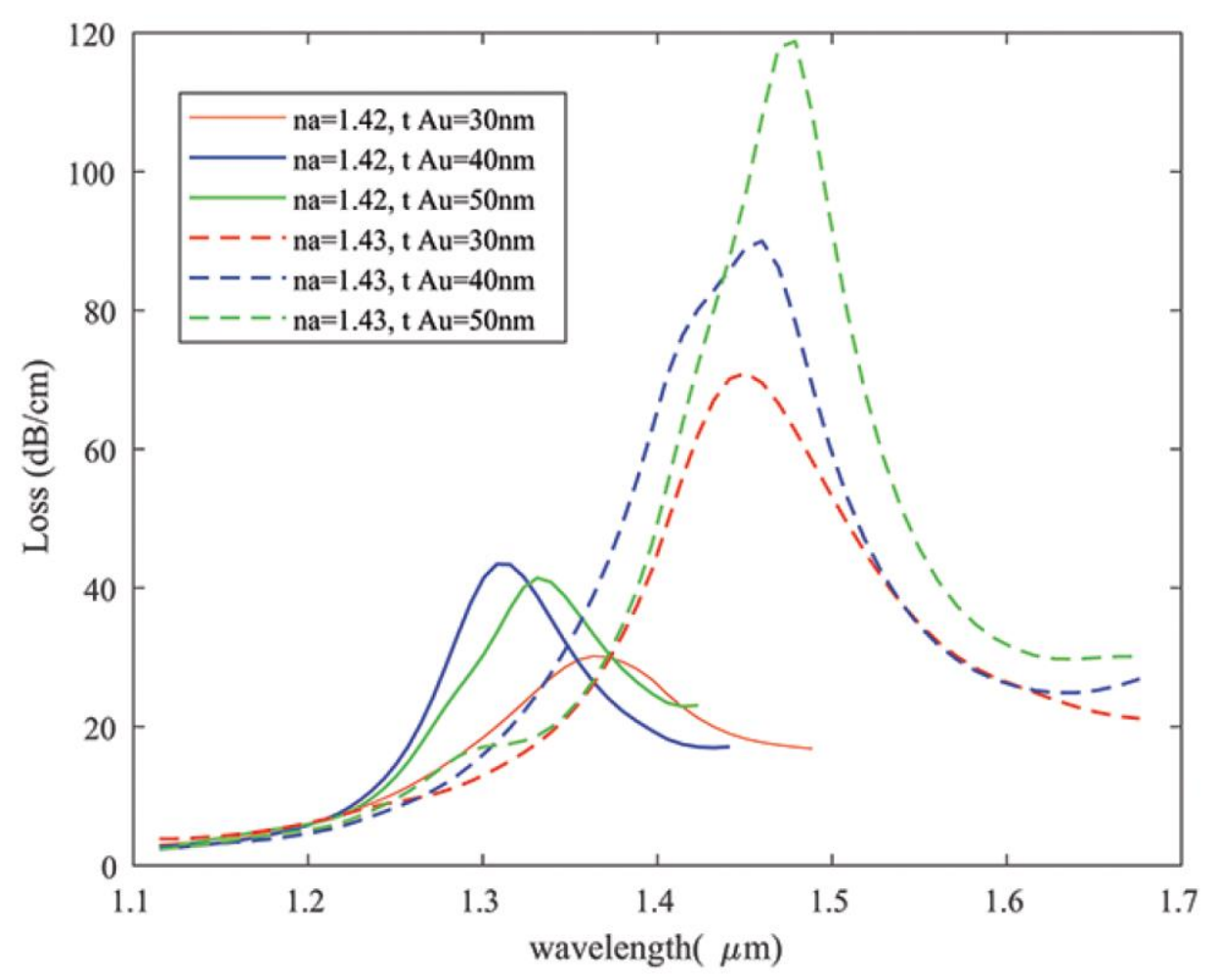

a

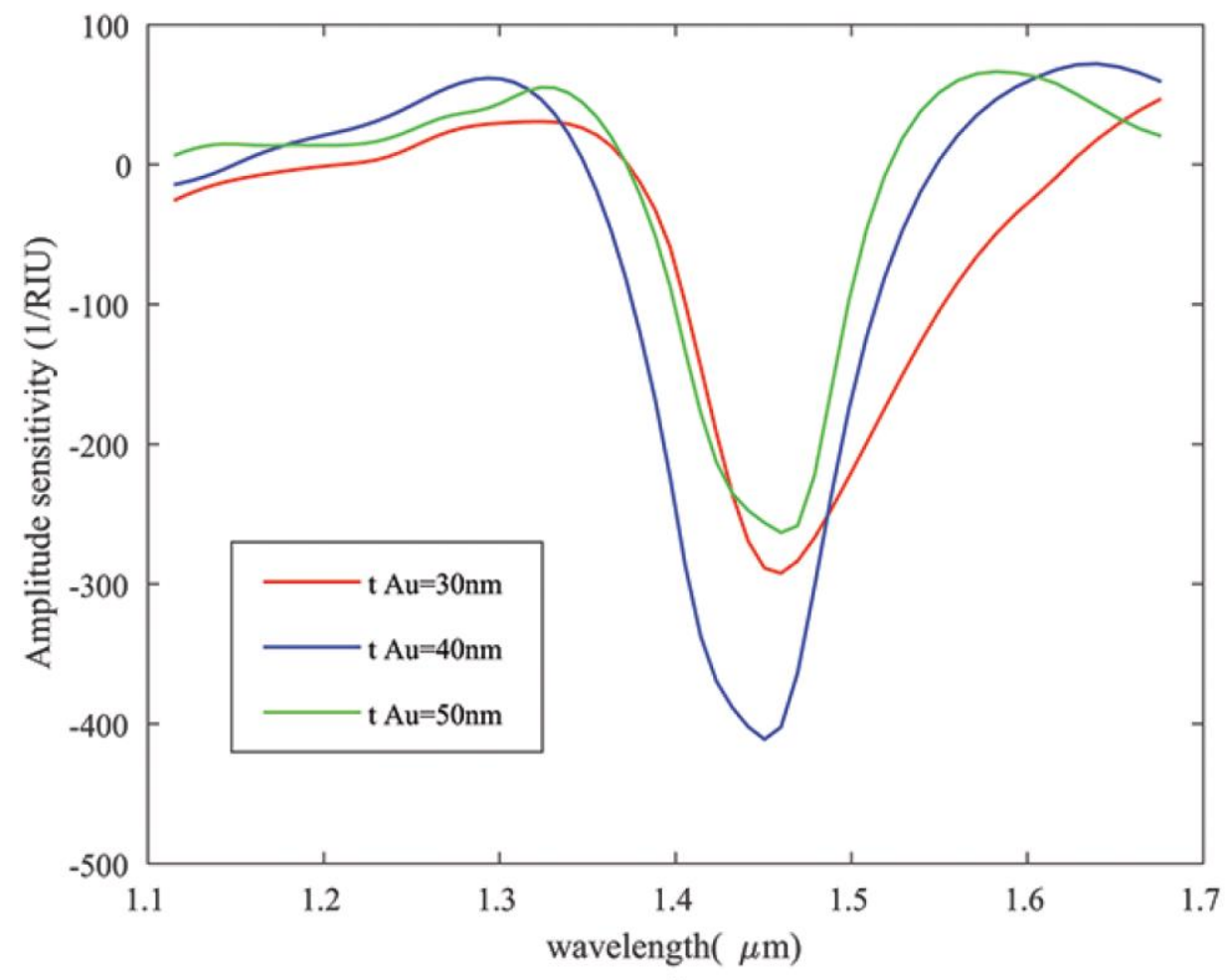

b 
Fig. 5 a Loss variation for different thickness of gold layer of the proposed sensor and $\mathbf{b}$ amplitude sensitivity for different thickness of gold layer with $t_{\mathrm{TiO}_{2}}=7 \mathrm{~nm}$

Generally, geometric parameters have a dominant effect on the sensor performance. The effect of changing of $A u$ and $\mathrm{TiO}_{2}$ thickness on loss spectra are depicted in figures 5 and 6, respectively. As it is seen from figure 5 (a) with increasing the gold thickness from 30 to $50 \mathrm{~nm}$, loss increases and wavelength redshifts. Panel (b) shows amplitude sensitivity; the maximum $S_{A}$ of $411.1 R I U^{-1}$ is obtained for $t_{A u}=40 \mathrm{~nm}$ with $n_{a}$ varying from 1.42 to 1.43 . This results summarized in table 1 which included both wavelength and amplitude sensitivity for proposed sensor with various thickness of gold. Considering wavelength and amplitude sensitivity, $t_{A u}=40 \mathrm{~nm}$ is chosen as optimum thickness of $A u$ in our calculations.

Table 1. Effect of gold thickness on the $S_{w}$ and $S_{A}$

\begin{tabular}{|c|c|c|}
\hline Au thickness $(\boldsymbol{n m})$ & Wavelength sensitivity $\left(\boldsymbol{S}_{\boldsymbol{w}}, \frac{n m}{R I U}\right)$ & Amplitude sensitivity $\left(\boldsymbol{S}_{A}, \frac{1}{R I U}\right)$ \\
\hline 30 & 8728 & 292.3 \\
\hline 40 & 15167 & 411.1 \\
\hline 50 & 14768 & 263.2 \\
\hline
\end{tabular}

By this value of $t_{A u}$, the effect of different $\mathrm{TiO}_{2}$ thicknesses on loss curves is illustrated in figure 6 where wavelength sensitivity can be calculated by its data.

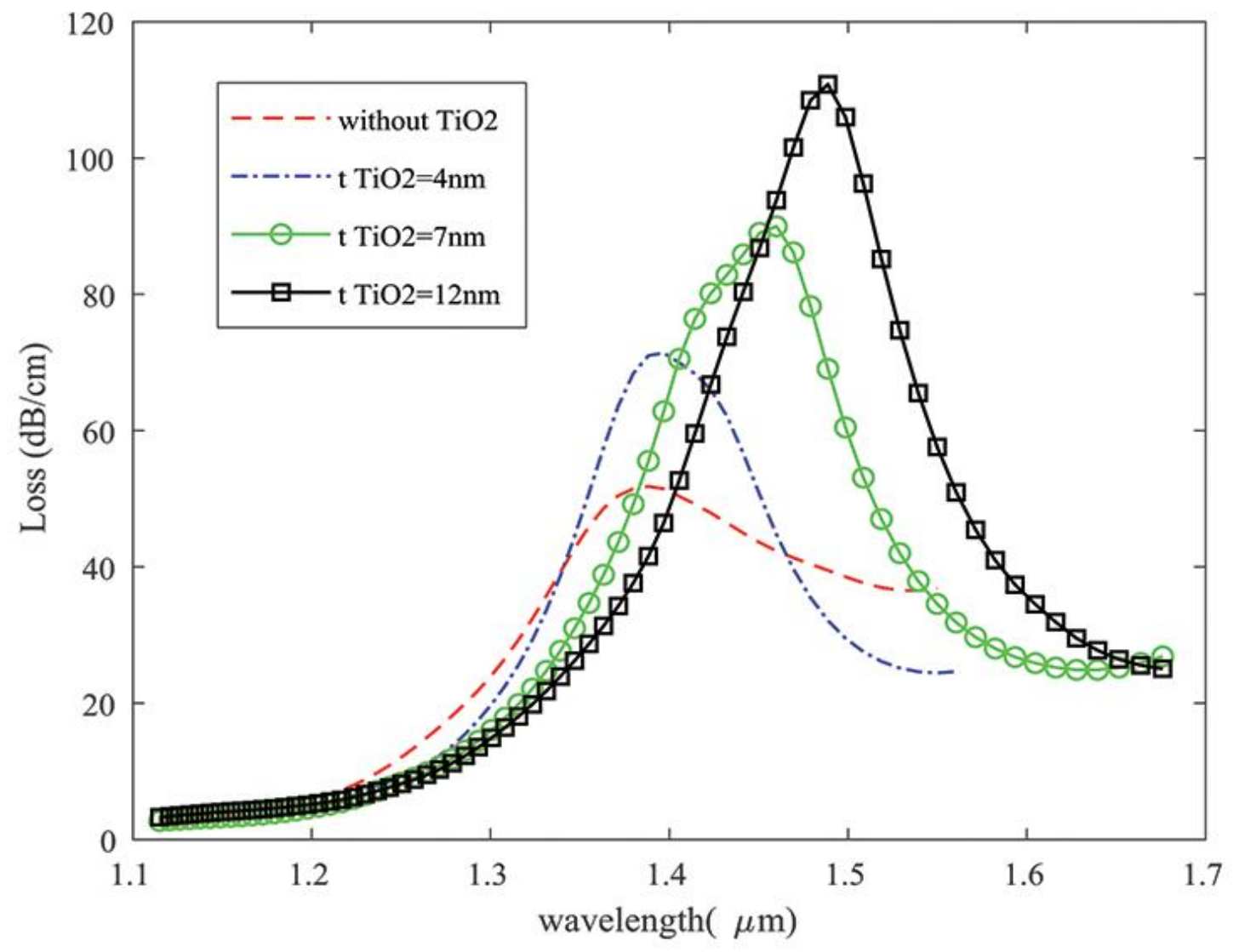


Fig. 6 Loss curves for thickness variation of $\mathrm{TiO}_{2}$ for $n_{a}=1.43, t_{A u}=40 \mathrm{~nm}$

The obtained $S_{W}$ for given $\mathrm{TiO}_{2}$ thicknesses are 13024, 12538, 15167, and $14157 \frac{\mathrm{nm}}{\mathrm{RIU}}$, respectively in which $t_{\mathrm{TiO}_{2}}=7 \mathrm{~nm}$ shows better sensitivity. Titanium dioxide has not only diminished adhesion problem but because of its high refractive index, it strongly attracts the field from the core mode, and causes strong coupling between core and plasmonic mode [21].

Besides of sensitivity, another factor for analyzing sensor performance is figure of merit (FOM) which can be defined as the ratio of sensitivity to full width at half maximum (FWHM) as [22]:

$F O M\left(R I U^{-1}\right)=\frac{\text { sensitivity }\left(\frac{n m}{R I U}\right)}{F W H M(n m)}$.

Now then, sensitivities and FOM of the proposed sensor are investigated for the wide range of analyte RI which are summarized in table 2.

Table 2. Performance analysis of the proposed sensor by varying the dielectric RI.

\begin{tabular}{|c|c|c|c|c|}
\hline Dielectric RI & $\boldsymbol{S}_{w}\left(\frac{n m}{R I U}\right)$ & $\boldsymbol{S}_{A}\left(\boldsymbol{R I U ^ { - 1 }}\right)$ & FOM $\left(\boldsymbol{R I U ^ { - 1 }}\right)$ & Resolution $(\boldsymbol{R I U})$ \\
\hline $1.42-1.43$ & 15167 & 411.1 & 207.19 & $6.6 \times 10^{-6}$ \\
\hline $1.43-1.44$ & 6894 & 314.5 & 62.55 & $1.4 \times 10^{-5}$ \\
\hline $1.44-1.45$ & 3158 & 59.95 & 35.40 & $3.1 \times 10^{-5}$ \\
\hline $1.45-1.46$ & 2179 & 19.8 & 22.91 & $4.6 \times 10^{-5}$ \\
\hline
\end{tabular}

The proposed sensor shows the best performance in RI range of 1.42-1.43 with respect of wavelength and amplitude sensitivities and FOM. By increasing of $n_{a}$, these sensing factors decrease monotonically.

Resolution of the sensor is also essential to determine detection capability of offered sensor and can be computed by [22]:

$R(R I U)=\Delta n_{a} \times \Delta \lambda_{\text {min }} / \Delta \lambda_{\text {peak }}$,

where $\Delta \lambda_{\min }$ is assumed to be $0.1 \mathrm{~nm}$. The maximum resolution of proposed sensor is obtained as high as $6.6 \times 10^{-6}$. Therefore the smallest change in analyte IR in order of $10^{-6}$ can be detected with a high degree of accuracy.

Last part of this work devoted to effect of grating on the surface of structure corresponding to the second configuration, figure 1 (b). A similar calculation are done for this configuration in the presence of grating for various structural parameters such as segment number, segmented metal film thickness and thickness of continuum part of metal layer. A typical SPR mode profile for grated dual-core PCF-SPR sensor is presented in figure 7. Simultaneously localized SPR and propagated SPR in the neighborhood of segment part are clearly observed. 


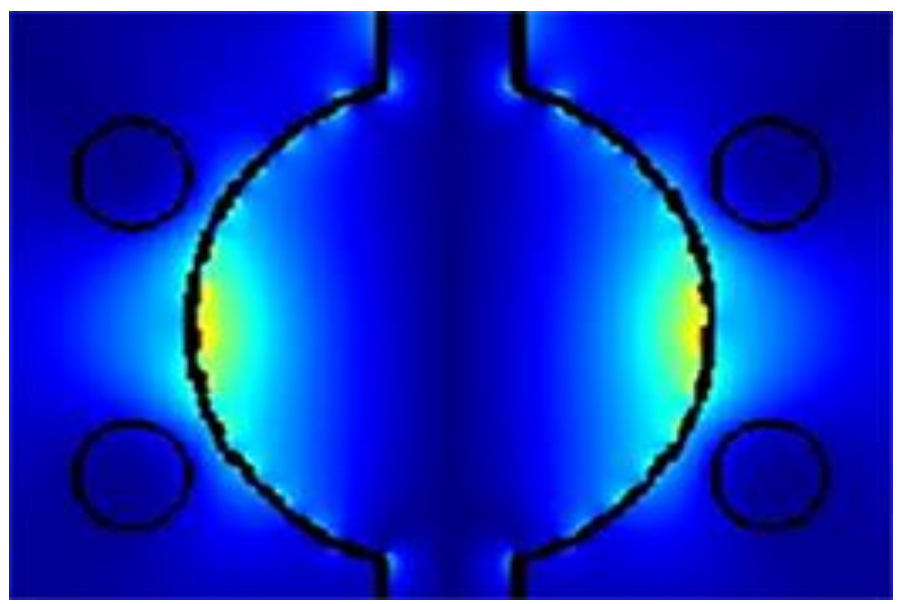

Fig. 7 Electric field distribution of the SPR mode for grated structure for $n_{a}=1.43$

The wavelength sensitivity, amplitude sensitivity and figure of merit for grated structure are computed and tabulated for different thicknesses of $\mathrm{TiO}_{2}$ and also in absence of $\mathrm{TiO}_{2}$ layer. Evidently, as shown in table 3, in all case of the presence of extra $\mathrm{TiO}_{2}$ layer, the proposed sensor shows better results in comparison with the bare one, the absence of $\mathrm{TiO}_{2}$ layer. Thus, $\mathrm{TiO}_{2}$ has the definite effect on improving sensor detection sensitivity. It is seen that the maximum values of $S_{W}, S_{A}$, and FOM belong to grating configuration associated with $5 \mathrm{~nm}$ of $\mathrm{TiO}_{2}$ thickness. Therefore, we continue our simulation with $5 \mathrm{~nm}$ thickness of $\mathrm{TiO}_{2}$.

Table 3. Performance analysis of the proposed sensor for grating configuration by varying of $\mathrm{TiO}_{2}$ thickness.

\begin{tabular}{|c|c|c|c|}
\hline $\boldsymbol{t}_{\mathrm{TiO}_{2}}$ & Maximum $\boldsymbol{S}_{W},\left(\frac{\mathrm{nm}}{\mathrm{RIU}}\right)$ & Maximum $\boldsymbol{S}_{\boldsymbol{A}},\left(\frac{\mathbf{1}}{\mathrm{RIU}}\right)$ & $\boldsymbol{F O M},\left(\frac{\mathbf{1}}{\boldsymbol{R I U}}\right)$ \\
\hline $0 \mathrm{~nm}$ & 12397 & 302.9 & 84.91 \\
\hline $3 \mathrm{~nm}$ & 12068 & 408.4 & 150.85 \\
\hline $5 \mathrm{~nm}$ & 13295 & 511.4 & 166.18 \\
\hline $6 \mathrm{~nm}$ & 9116 & 320.8 & 99.08 \\
\hline $7 \mathrm{~nm}$ & 12638 & 260.8 & 133.03 \\
\hline $10 \mathrm{~nm}$ & 11931 & 326.9 & 80.07 \\
\hline
\end{tabular}

The sensing parameters for different analyte refractive index of grated structure with $t_{\mathrm{TiO}_{2}}=5 \mathrm{~nm}, t_{A u}=40 \mathrm{~nm}$ and segment number of 28 are came up in table 4 . It can be concluded from this table that the $S_{W}, S_{A}$ and FOM reach their maximum value when IR varies between 1.43-1.44.

Table 4. Performance analysis of the proposed sensor for grating configuration by varying the dielectric RI.

\begin{tabular}{|c|c|c|c|c|}
\hline Dielectric RI & $\lambda_{\text {peak }}(\boldsymbol{\mu m})$ & $\boldsymbol{S}_{w}\left(\frac{n m}{R I U}\right)$ & $\boldsymbol{S}_{\boldsymbol{A}}\left(\boldsymbol{R I U ^ { - 1 }}\right)$ & $F O M\left(\boldsymbol{R I U}^{-\mathbf{1}}\right)$ \\
\hline 1.42 & 1.4046 & 5790 & 234.9 & 75.19 \\
\hline 1.43 & 1.4625 & 13295 & 511.4 & 166.18 \\
\hline 1.44 & 1.5954 & 6282 & 124.5 & 73.90 \\
\hline 1.45 & 1.6582 & 2655 & 16.27 & 39.04 \\
\hline 1.46 & 1.6848 & - & - & - \\
\hline
\end{tabular}

Figure 8 shows the effect of $\mathrm{N}$, segment number, on the wavelength sensitivity with and without $\mathrm{TiO}_{2}$ layer. Also in table 5 the effect of this parameter on the other sensing factors are tabulated. 


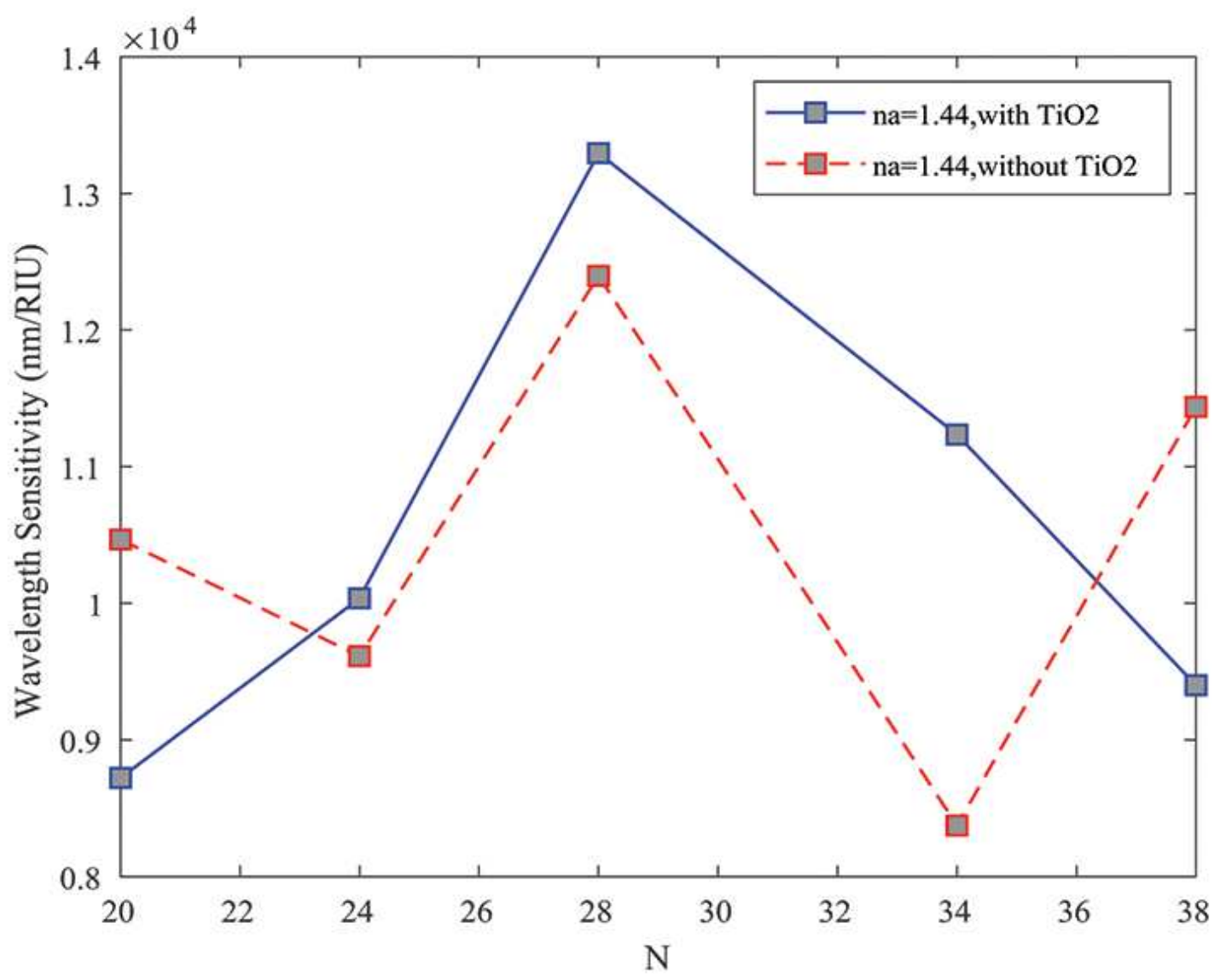

Fig. 8 Wavelength sensitivity of the core mode for different $\mathrm{N}$ for $n_{a}=1.43$

This figure depicted that the existence of $\mathrm{TiO}_{2}$ layer has considerable role on $S_{w}$ behavior. In fact, in the absence of $\mathrm{TiO}_{2}$, when $\mathrm{N}$ increases, sensitivity varies in a zigzag form where it has smooth behavior with a single peak value in the presence of $\mathrm{TiO}_{2}$. Peak value in both cases occurs in $N=28$. It is worth mentioned that, the similar calculation for $\mathrm{N}$ values smaller than 20 is done which gives sensitivities lower than the obtained peak value. Consequently the sensitivity can be effectively tuned by the segment number. We consider $N=28$ as an optimized segment number.

Table 5. Effect of $\mathrm{Au}$ grated with $5 \mathrm{~nm}$ thickness of $\mathrm{TiO}_{2}$ on $S_{w}, S_{A}, \mathrm{FOM}$ and average sensitivity for different $\mathrm{N}$.

\begin{tabular}{|c|c|c|c|c|c|c|c|c|}
\hline \multirow{2}{*}{$\begin{array}{l}\text { Segment } \\
\text { number } N\end{array}$} & \multicolumn{2}{|c|}{ maximum $\boldsymbol{S}_{\boldsymbol{w}}\left(\frac{\mathrm{nm}}{\boldsymbol{R} I \boldsymbol{U}}\right)$} & \multicolumn{2}{|c|}{ maximum $\boldsymbol{S}_{A}\left(\boldsymbol{R I} \boldsymbol{U}^{-\mathbf{1}}\right)$} & \multicolumn{2}{|c|}{ maximum FOM $\left(\boldsymbol{R I U} \boldsymbol{U}^{-\mathbf{1}}\right)$} & \multicolumn{2}{|c|}{ Average sensitivity } \\
\hline & Without $\mathrm{TiO}_{2}$ & with $\mathrm{TiO}_{2}$ & Without $\mathrm{TiO}_{2}$ & with $\mathrm{TiO}_{2}$ & Without & with $\mathrm{TiO}_{2}$ & Withou & with $\mathrm{TiO}_{2}$ \\
\hline 20 & 10467 & 8722 & 285.4 & 301.3 & 73.71 & 96.91 & 5026 & 4899.3 \\
\hline 24 & 9613 & 10036 & 273.4 & 409.8 & 99.10 & 145.44 & 6101.6 & 6525.6 \\
\hline 28 & 12397 & 13295 & 302.9 & 511.4 & 130.5 & 166.18 & 8193 & 8455.6 \\
\hline 34 & 8373 & 11235 & 283.4 & 472.5 & 64.90 & 160.5 & 5501.3 & 7521.6 \\
\hline 38 & 11438 & 9400 & 225.1 & 400 & 105.9 & 110.58 & 7503.3 & 6862.6 \\
\hline
\end{tabular}


From table 5, it can be observed that when $\mathrm{N}$ varies from 20 to 38, the maximum obtained wavelength and amplitude sensitivity, FOM, and also average sensitivity are $13295 \frac{\mathrm{nm}}{R I U}, 511.4 R^{-1} U^{-1}, 166.18 R I U^{-1}$, and $8455.6 \frac{\mathrm{nm}}{R I U}$ in presence of $\mathrm{TiO}_{2}$ layer. The corresponding values decrease to $12397 \frac{\mathrm{nm}}{\mathrm{RIU}}, 302.9 \mathrm{RIU}^{-1}, 130.5 \mathrm{RIU} U^{-1}, 8193 \frac{\mathrm{nm}}{\mathrm{RIU}}$ in absence of $\mathrm{TiO}_{2}$. It is worth noting that these maximum values allocate to $\mathrm{N}=28$.

Considering the optimized values of $t_{A u}=40 \mathrm{~nm}, t_{T i O_{2}}=5 \mathrm{~nm}$, and $N=28$, the effect of $d_{1}$ and $d_{2}$ on the sensor performance is considered simultaneously, with the condition of $d_{1}+d_{2}=40 \mathrm{~nm},\left(d_{1}+d_{2}=t_{A u}\right)$. Results are illustrated in figure 9 which shows the loss spectra for different arrangement of $d_{1}$ and $d_{2}$. For arrangement of $d_{1}=30 \mathrm{~nm}$ and $d_{2}=10 \mathrm{~nm}$ when $n_{a}$ changes from 1.42 to 1.45 , the wavelength sensitivities are $7432,10467,5805$, $3205 \frac{\mathrm{nm}}{\mathrm{RIU}}$, while the corresponding values are $6379,12397,5805 \frac{\mathrm{nm}}{\mathrm{RIU}}$ for the arrangement of $d_{1}=25 \mathrm{~nm}$ and $d_{2}=$ $15 \mathrm{~nm}$.

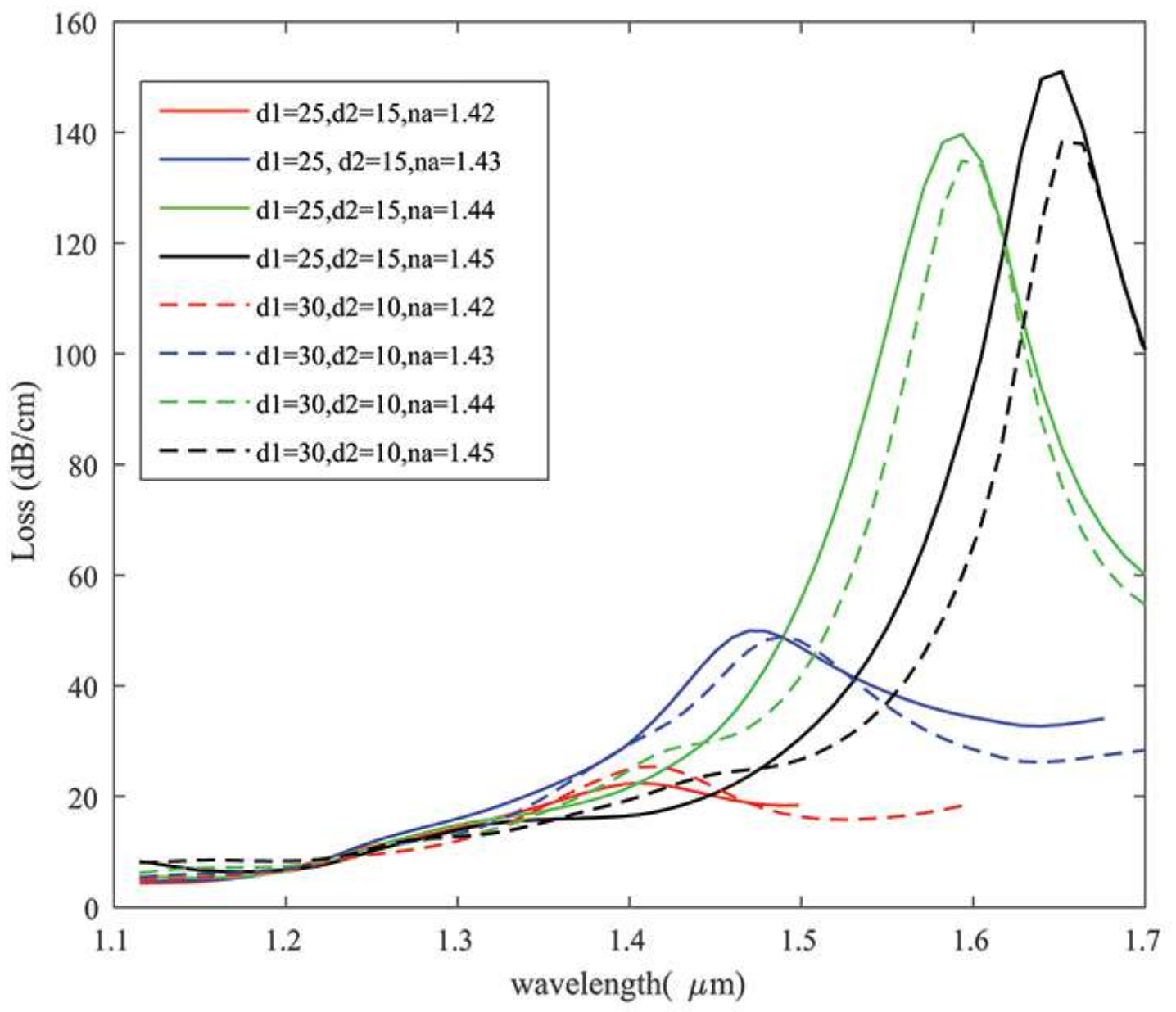

Fig. 9 Loss spectra as a function of different arrangement of $d_{1}$ and $d_{2}$

As a result, when $d_{1}=25 \mathrm{~nm}$ and $d_{2}=15 \mathrm{~nm}$ the maximum wavelength sensitivity is higher than that of when the sensor is set with $d_{1}=30 \mathrm{~nm}$ and $d_{2}=10 \mathrm{~nm}$.

\section{Conclusion}


In summary, two different configuration of highly sensitive opening-up dual-core photonic crystal fiber sensors based on surface plasmon resonance have been introduced and numerical analyses have been performed by using of FDTD method. The opening up structure not only simplifies analyte infiltering and gold coating but also offers the capacity for real-time sensing. The results reveal that the odd mode for y-polarization coupled with odd SPR mode more strongly due to its largest confinement loss in two structures. Additionally, a comparison was made in each configuration with presence of $\mathrm{TiO}_{2}$ and in absence of $\mathrm{TiO}_{2}$ in terms of the sensitivity and FOM. As regards applying $\mathrm{TiO}_{2}$ layer improve the sensitivity about $16 \%$ for first configuration and about $7 \%$ for second configuration it was observed that dual-core SPR-PCF sensor with $\mathrm{TiO}_{2}$ thin layer without grating structure shows the highest sensing performance. Surprisingly this sensor has capability to detect higher or lower RI than the RI of the background material. Owning to highly sensitive response, the proposed sensor can be considered ideal for refractive index detection.

\section{Funding}

No funds, grants, or other support was received.

\section{Conflict of interest}

The authors have no conflicts of interest to declare that are relevant to the content of this article.

\section{Availability of data}

The datasets generated during the analysis of current study are available from the corresponding author on reasonable request.

\section{Author contributions}

Soghra Ghahramani: Conceptualization, Methodology, Formal analysis and investigation, Writing - original draft preparation.

Jamal Barvestani:, Supervision, Project administration, Writing - review and editing.

Bahar Meshginqalam: Writing - review and editing.

\section{Ethics approval}

This study is not involved human participants, their data or biological material.

\section{Consent to participate}

Informed consent was obtained from all individual participants included in the study.

\section{Consent to publish}

Authors give consent for the publication of identifiable details within the text to be published in the Plasmonics journal.

\section{References}

1. Zhao, Y., et al., Current status of optical fiber biosensor based on surface plasmon resonance. Biosensors and Bioelectronics, 2019. 142: p. 111505.

2. Wang, J., et al., Surface plasmon resonance sensor based on coupling effects of dual photonic crystal fibers for low refractive indexes detection. Results in Physics, 2020. 18: p. 103240.

3. Isti, M.I.A., et al., Asymmetrical D-channel photonic crystal fiber-based plasmonic sensor using the wavelength interrogation and lower birefringence peak method. Results in Physics, 2020. 19: p. 103372. 
4. Liu, C., et al., Near-infrared surface plasmon resonance sensor based on photonic crystal fiber with big open rings. Optik, 2020. 207: p. 164466.

5. Biplob Hossain, M., et al., Numerical development of high performance quasi D-shape PCF-SPR biosensor: An external sensing approach employing gold. Results in Physics, 2020. 18: p. 103281.

6. Paul, A.K., et al., An air-core photonic crystal fiber based plasmonic sensor for high refractive index sensing. Optics Communications, 2020. 464: p. 125556.

7. Chen, N., et al., Highly Sensitive Plasmonic Sensor Based on a Dual-Side Polished Photonic Crystal Fiber for Component Content Sensing Applications. Nanomaterials, 2019. 9(11).

8. Rifat, A.A., et al., Highly sensitive selectively coated photonic crystal fiber-based plasmonic sensor. Optics Letters, 2018. 43(4): p. 891-894.

9. Hasan, M.R., et al., Spiral Photonic Crystal Fiber-Based Dual-Polarized Surface Plasmon Resonance Biosensor. IEEE Sensors Journal, 2018. 18(1): p. 133-140.

10. Paul, A.K., A.K. Sarkar, and A. Khaleque, Dual-Core Photonic Crystal Fiber Plasmonic Refractive Index Sensor: A Numerical Analysis. Photonic Sensors, 2019. 9(2): p. 151-161.

11. Han, H., et al., A Large Detection-Range Plasmonic Sensor Based on An H-Shaped Photonic Crystal Fiber. Sensors, 2020. 20(4).

12. Haque, E., et al., Highly Sensitive Dual-Core PCF Based Plasmonic Refractive Index Sensor for Low Refractive Index Detection. IEEE Photonics Journal, 2019. 11(5): p. 1-9.

13. Haque, E., et al., Surface Plasmon Resonance Sensor Based on Modified D-Shaped Photonic Crystal Fiber for Wider Range of Refractive Index Detection. IEEE Sensors Journal, 2018. PP: p. 1-1.

14. Luan, N., et al., Opening up dual-core microstructured optical fiber-based plasmonic sensor with large detection range and linear sensitivity. Optical Materials Express, 2019. 9(2): p. 819-825.

15. Luan, N. and J. Yao, Surface Plasmon Resonance Sensor Based On Exposed-Core Microstructured Optical Fiber Placed With A Silver Wire. IEEE Photonics Journal, 2016. 8(1): p. 1-8.

16. Singh, S. and Y.K. Prajapati, TiO2/gold-graphene hybrid solid core SPR based PCF RI sensor for sensitivity enhancement. Optik, 2020. 224: p. 165525.

17. Otupiri, R., E.K. Akowuah, and S. Haxha, Multi-channel SPR biosensor based on PCF for multi-analyte sensing applications. Optics Express, 2015. 23(12): p. 15716-15727.

18. DeVore, J.R., Refractive Indices of Rutile and Sphalerite. Journal of the Optical Society of America, 1951. 41(6): p. 416-419.

19. Hasan, M.R., et al., A Highly Sensitive Gold-Coated Photonic Crystal Fiber Biosensor Based on Surface Plasmon Resonance. Photonics, 2017. 4(1).

20. Li, T., et al., A Refractive Index Sensor Based on H-Shaped Photonic Crystal Fibers Coated with AgGraphene Layers. Sensors, 2020. 20(3).

21. Islam, M.S., et al., A Hi-Bi Ultra-Sensitive Surface Plasmon Resonance Fiber Sensor. IEEE Access, 2019. 7: p. 79085-79094.

22. Fang, H., et al., Research on photonic crystal fiber based on a surface plasmon resonance sensor with segmented silver-titanium dioxide film. Journal of the Optical Society of America B, 2020. 37(3): p. 736-744. 


\section{Figures}

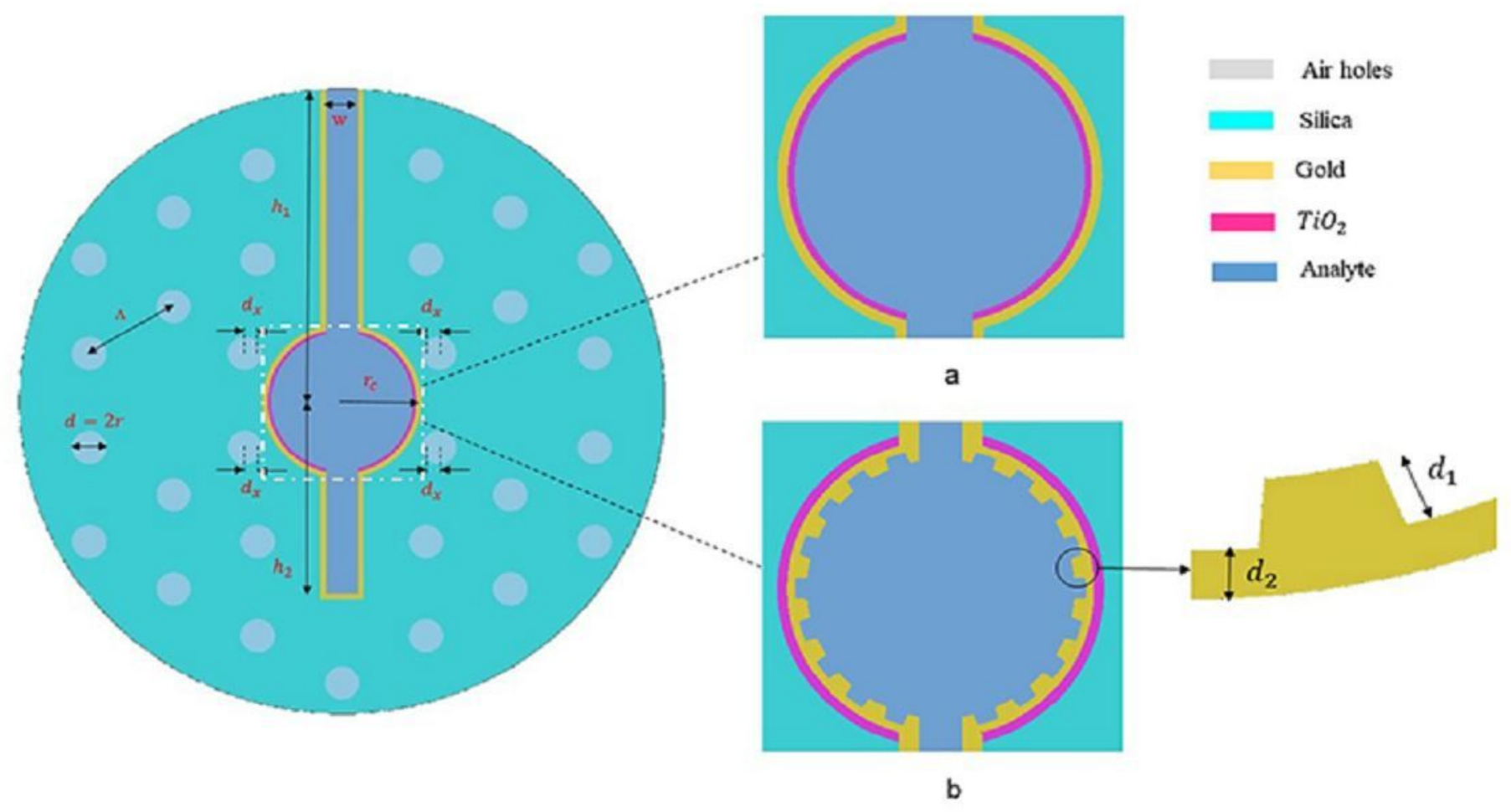

Figure 1

Cross-section view of DC-PCF-SPR sensor magnifying of central large hole a with Au and $\mathrm{QTTOO} \mathbb{Z}_{2} 2$ thin layer between $\mathrm{Au}$ and analyte $\mathrm{b}$ with Au nano-continues gratings layer and thin $\mathrm{QTiO} \_\_2$ layer between fiber material and $\mathrm{Au}$ 


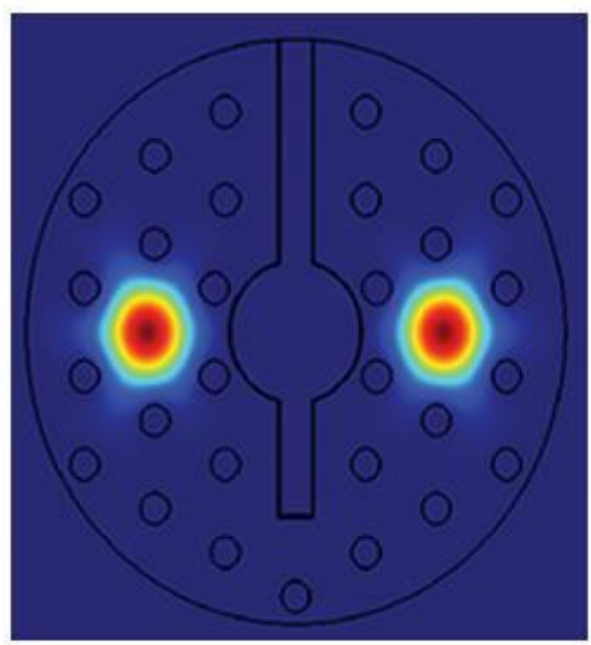

a

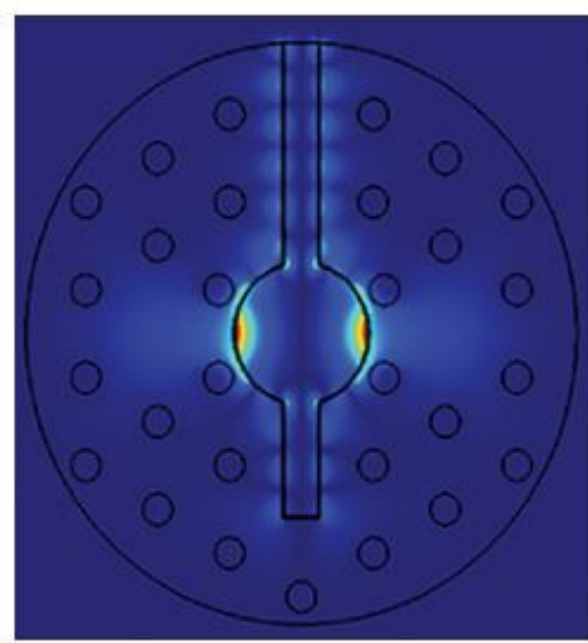

b

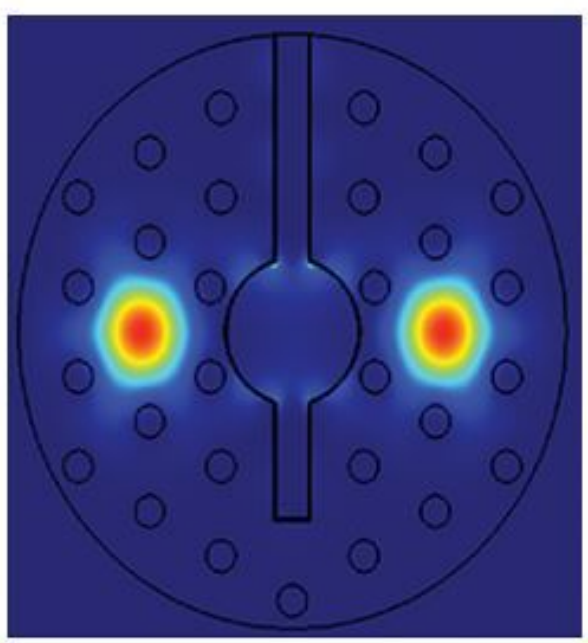

C

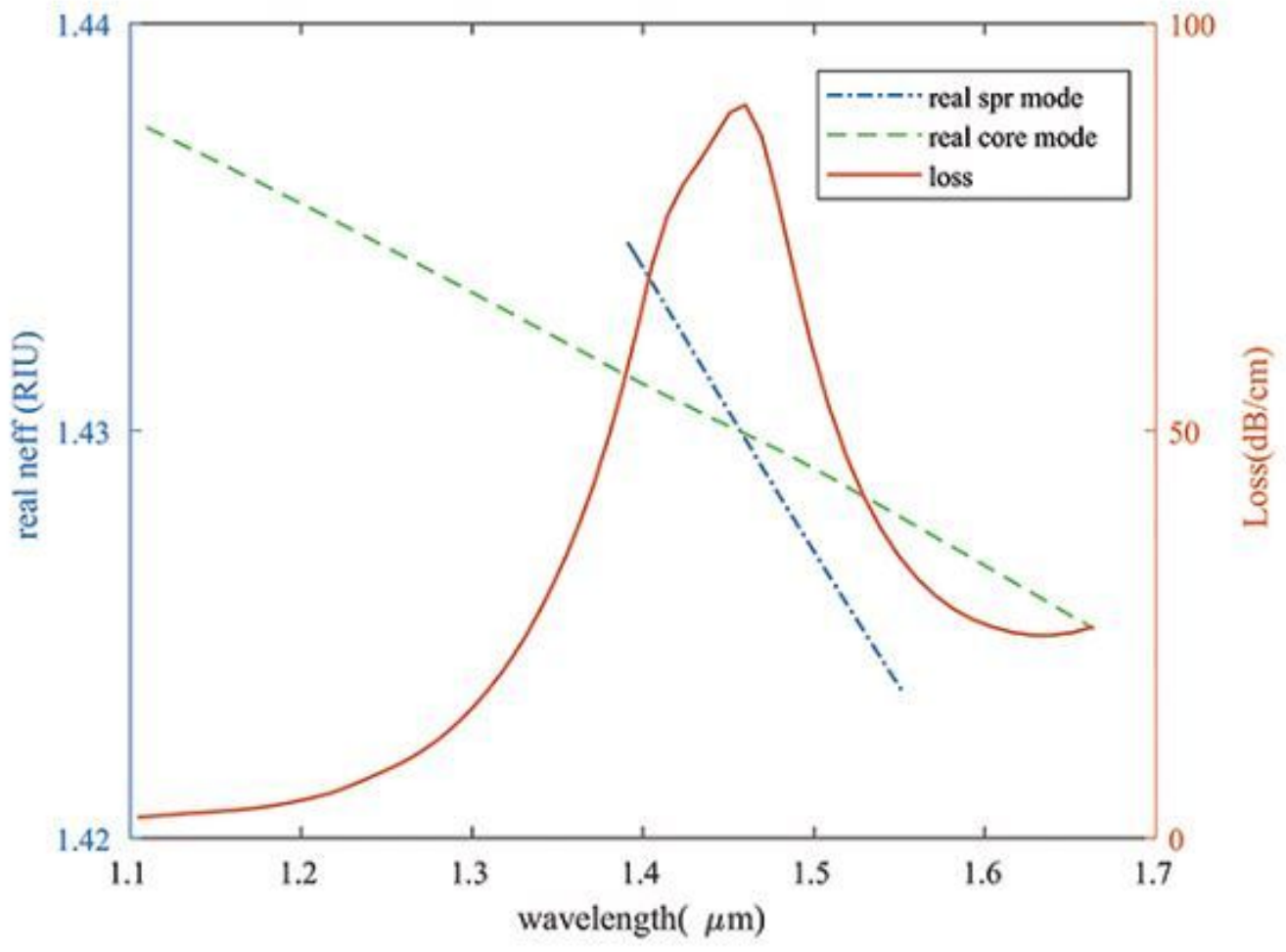

d

Figure 2

please see the manuscript file for the full caption 


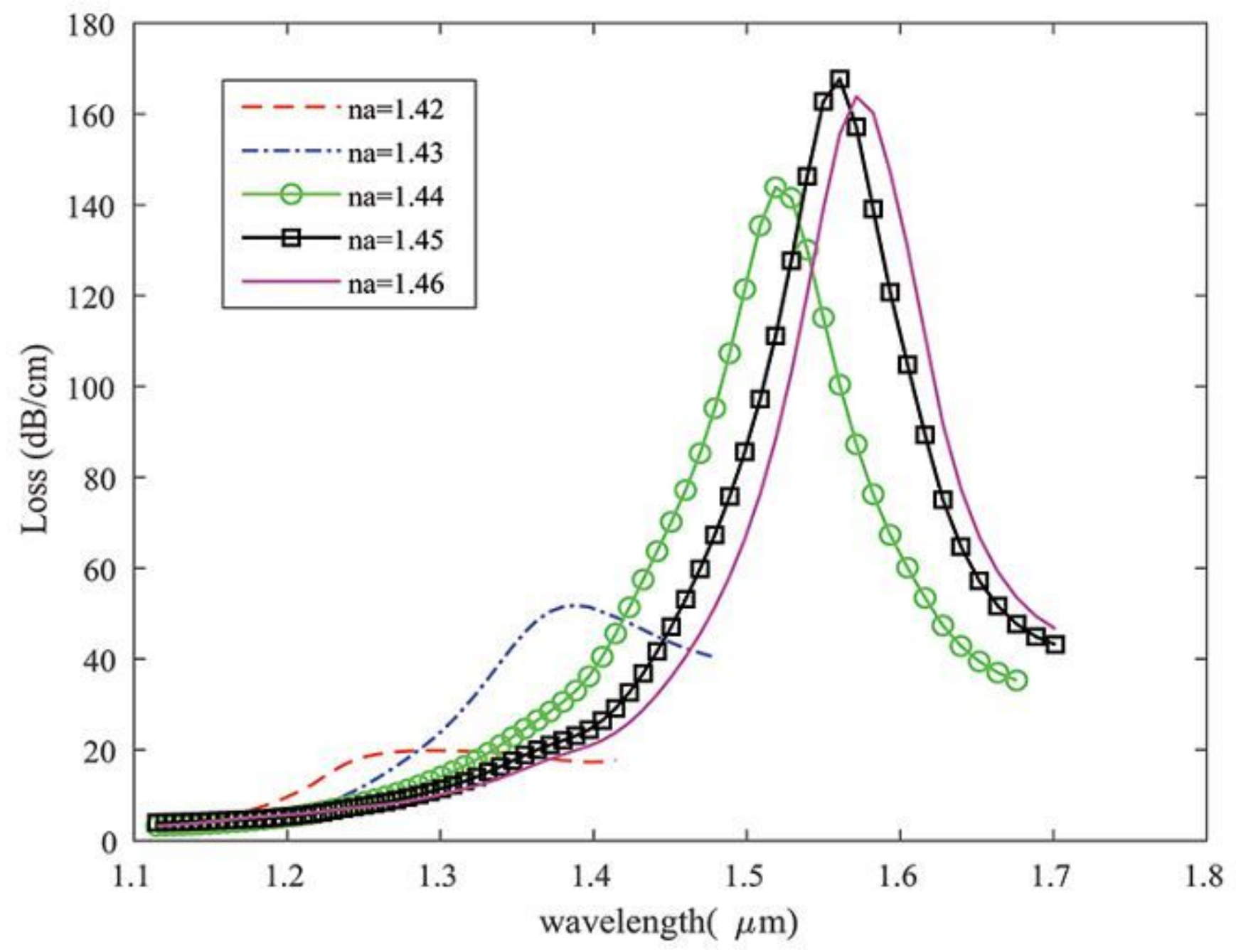

Figure 3

please see the manuscript file for the full caption 


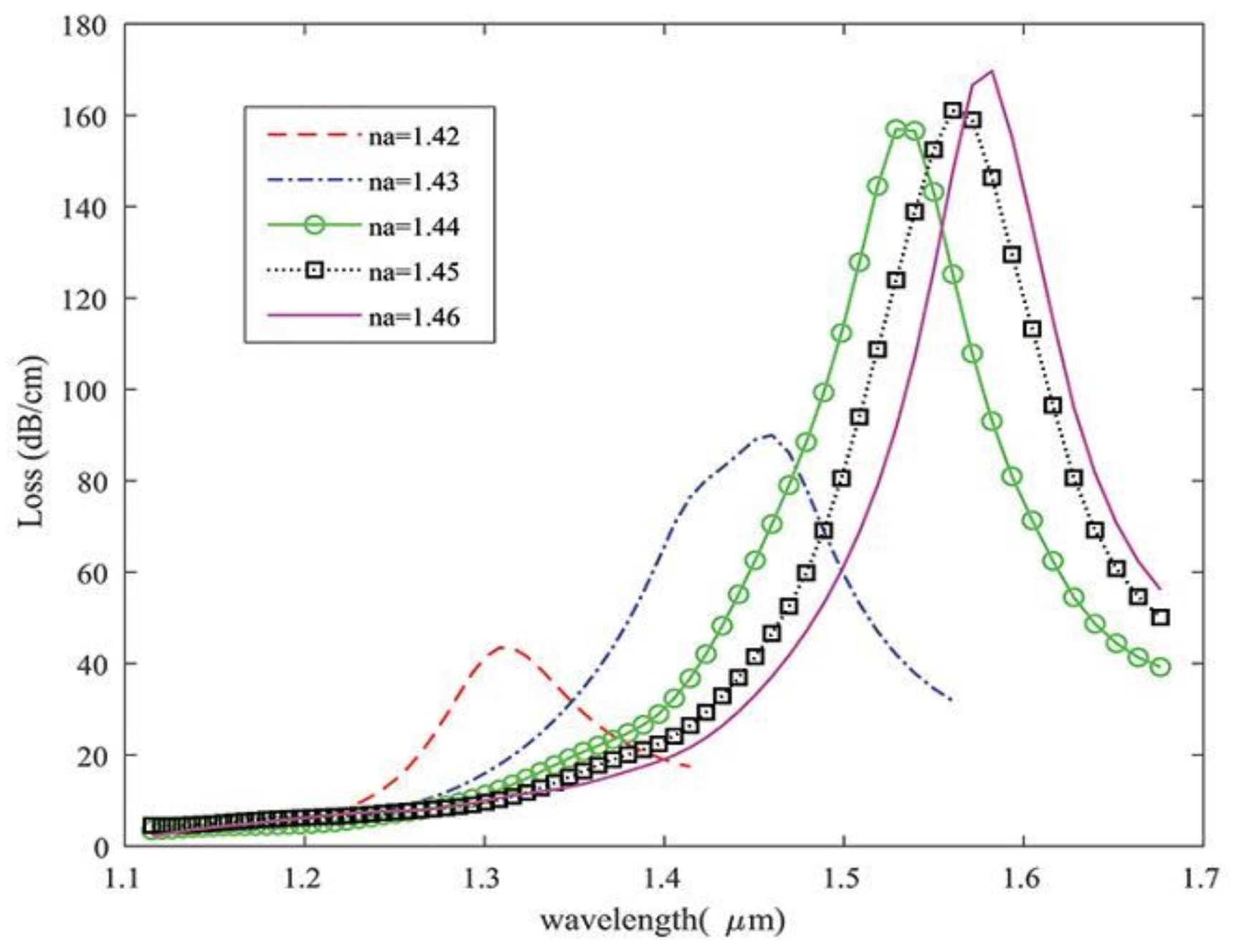

Figure 4

please see the manuscript file for the full caption 


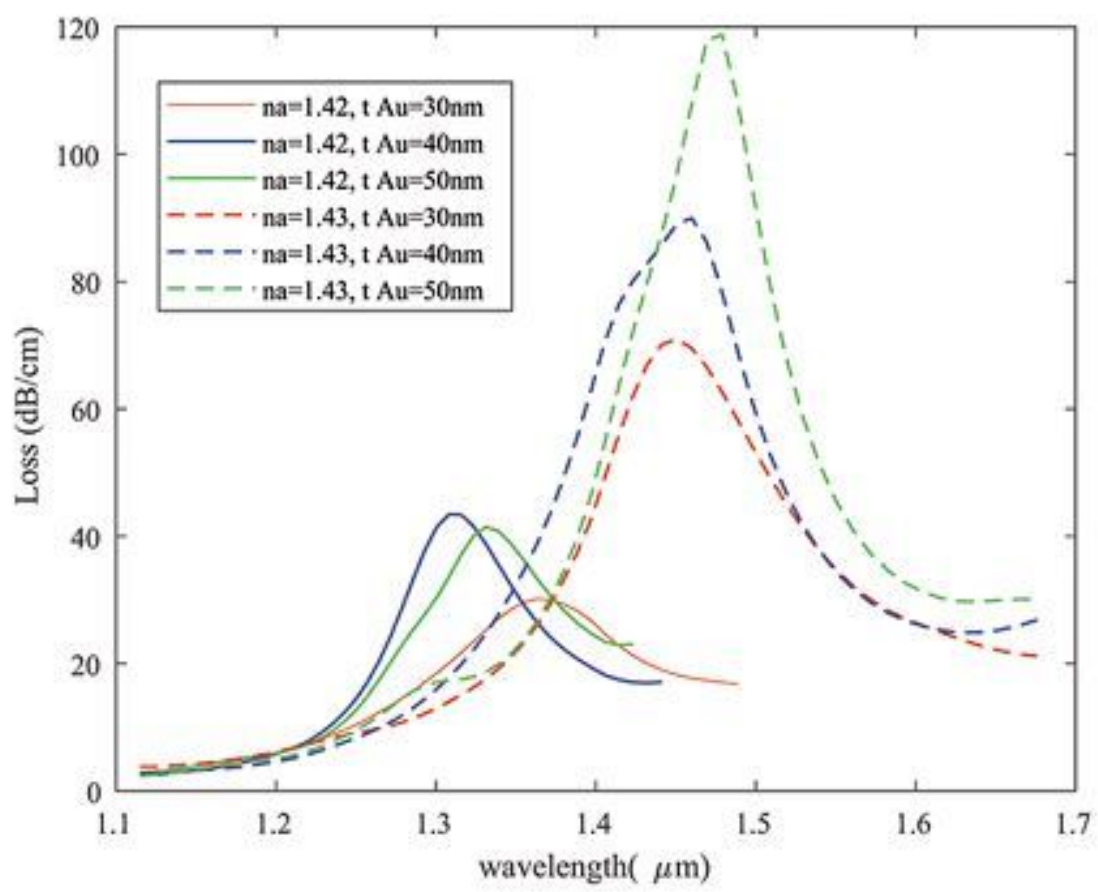

a

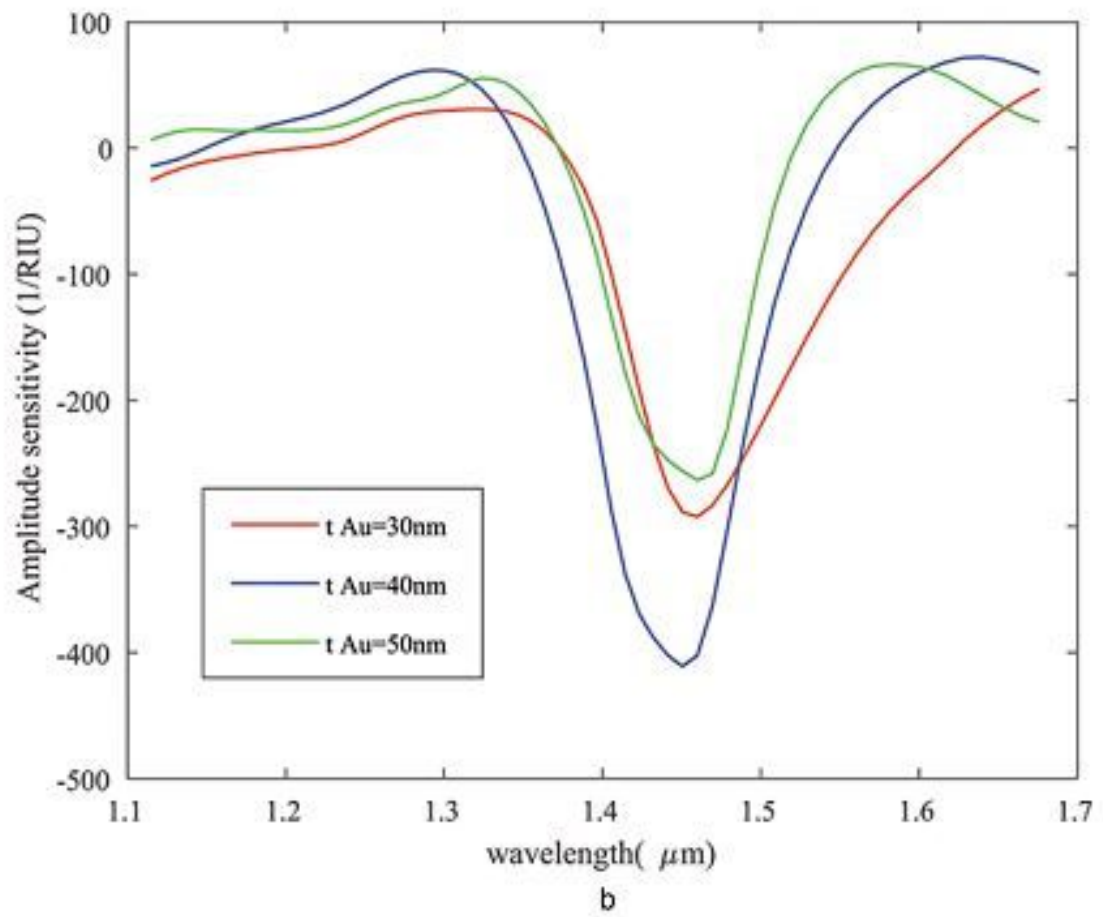

Figure 5

please see the manuscript file for the full caption 


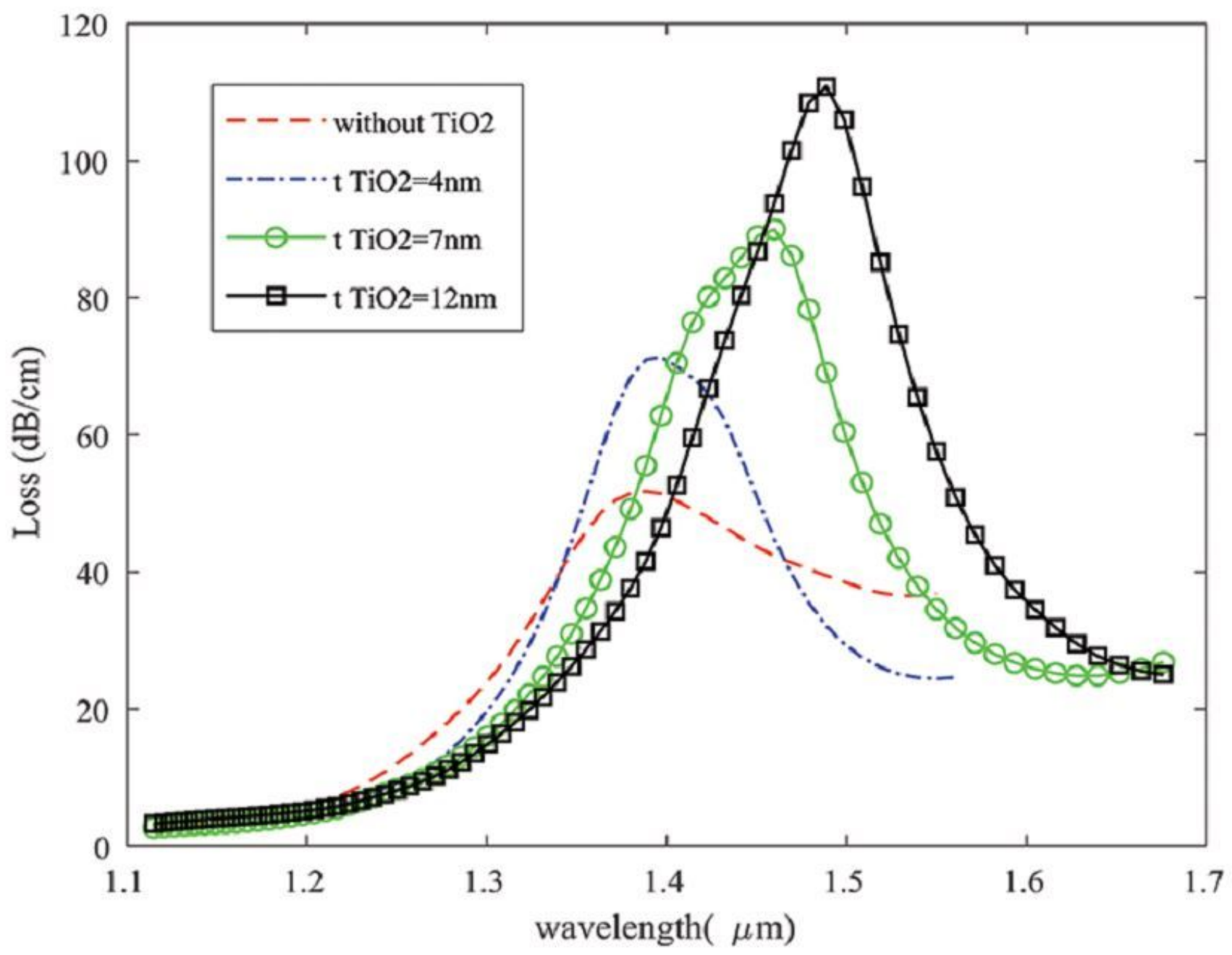

Figure 6

please see the manuscript file for the full caption 


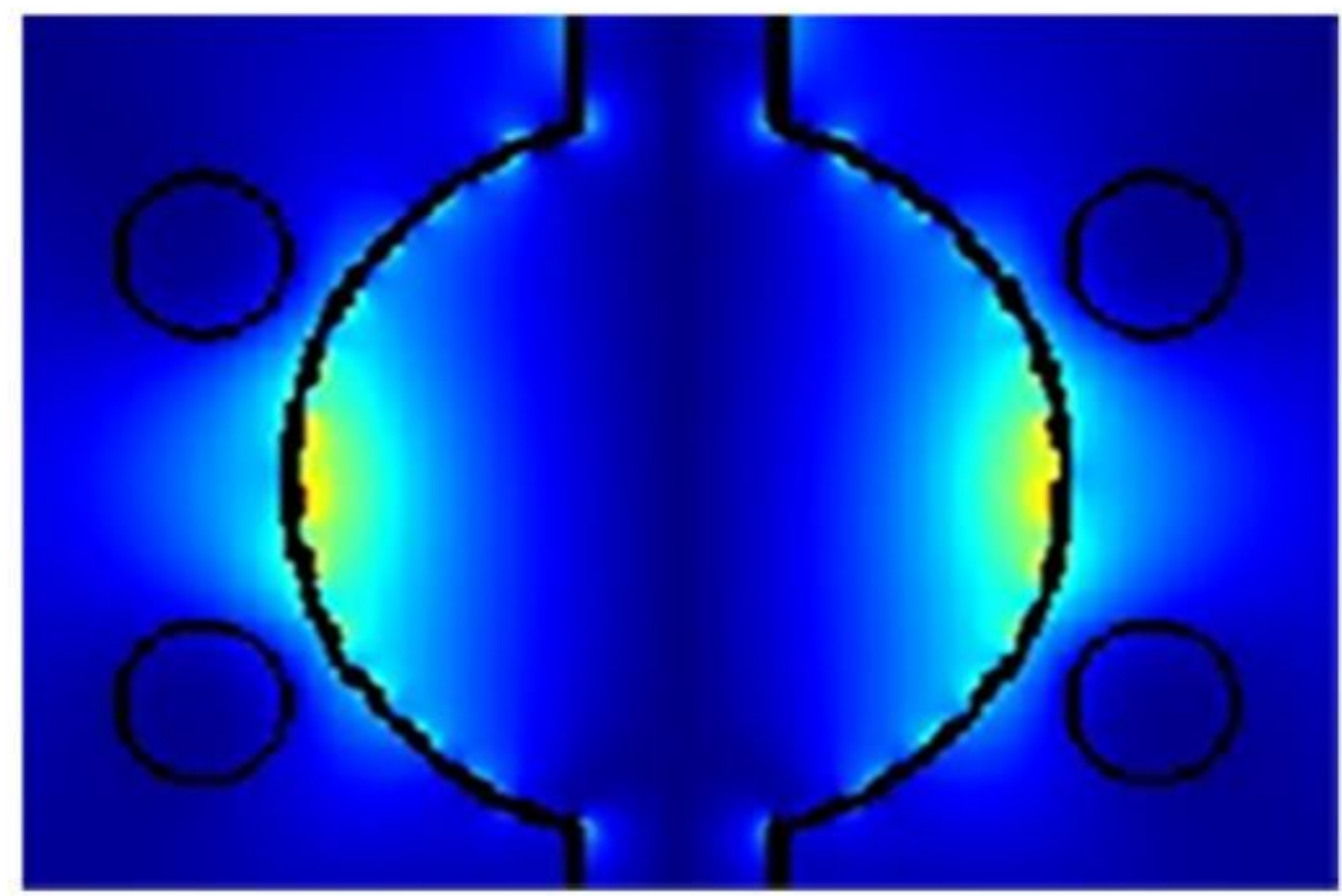

Figure 7

please see the manuscript file for the full caption 


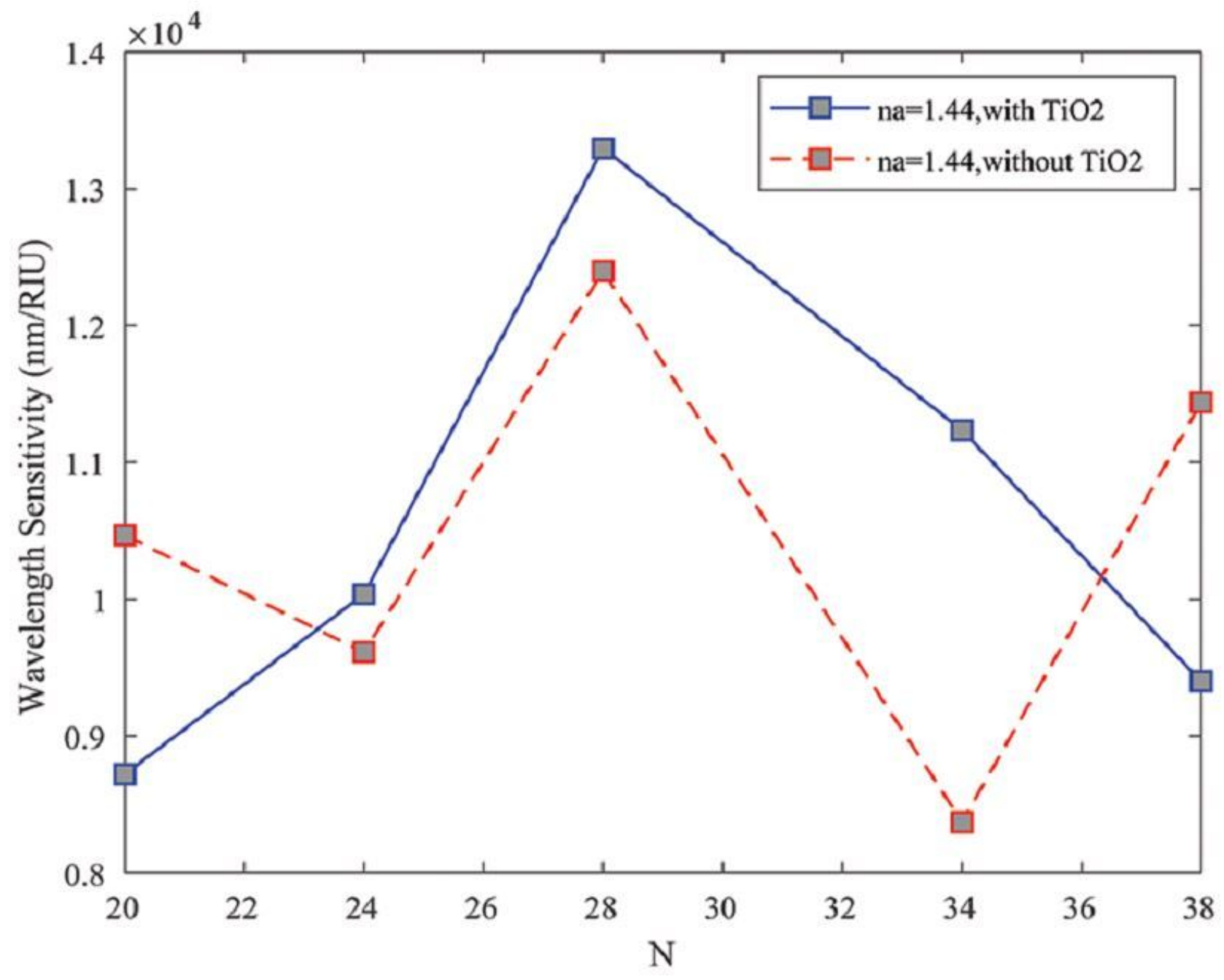

Figure 8

please see the manuscript file for the full caption 


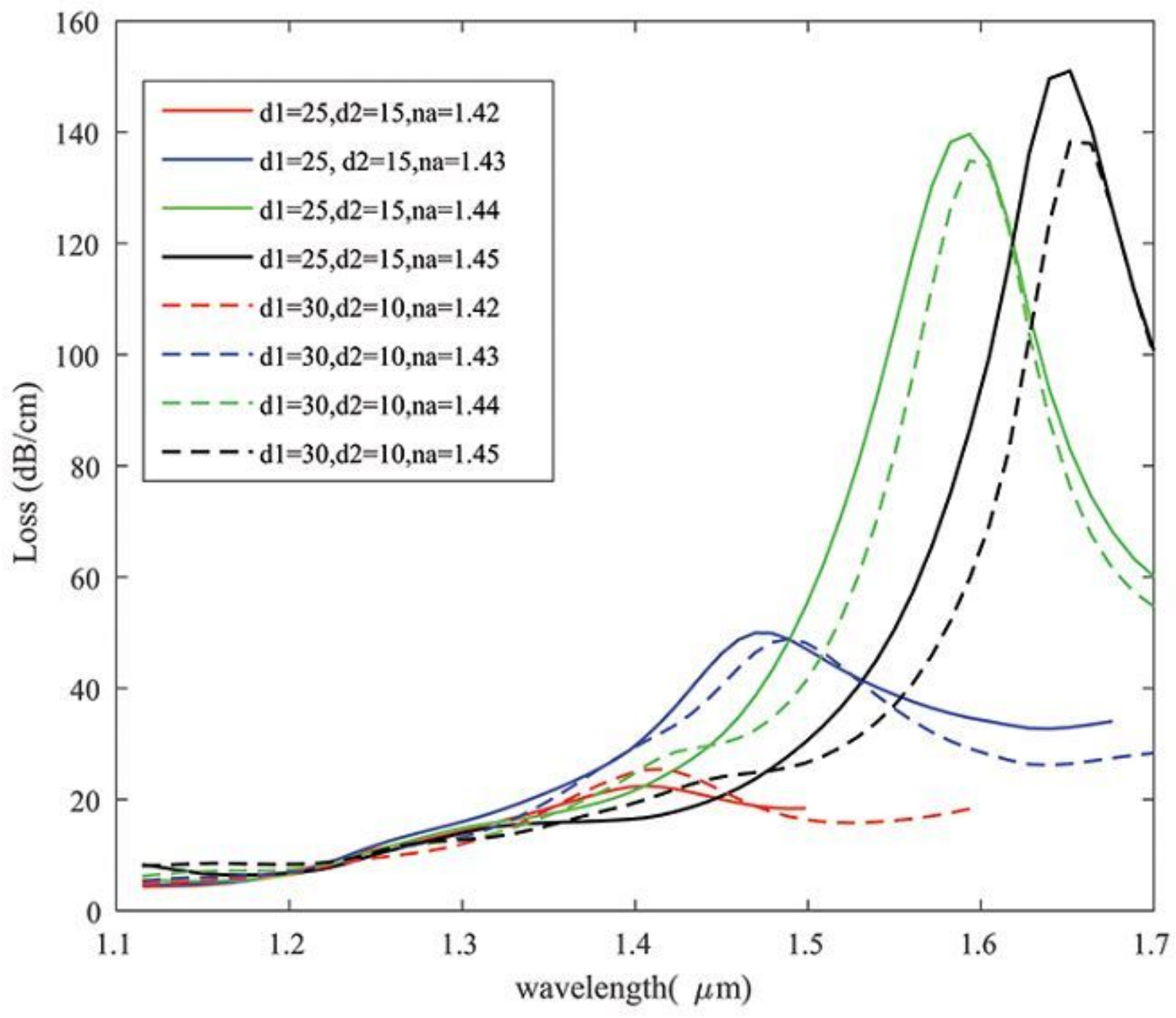

Figure 9

please see the manuscript file for the full caption 